\title{
Mevastatin blockade of autolysosome maturation stimulates LBH589-induced cell death in triple-negative breast cancer cells
}

\author{
Zhaohu Lin ${ }^{1,3}$, Zhuqing Zhang ${ }^{1}$, Xiaoxiao Jiang ${ }^{1}$, Xinhui Kou ${ }^{1}$, Yong Bao ${ }^{1}$, Huijuan \\ Liu $^{1}$, Fanghui Sun ${ }^{1}$, Shuang Ling ${ }^{2}$, Ning Qin ${ }^{3}$, Lan Jiang ${ }^{4}$, Yonghua Yang ${ }^{1}$ \\ ${ }^{1}$ Department of Pharmacology and Biochemistry, School of Pharmacy Fudan University, Shanghai 201203, China \\ ${ }^{2}$ Interdisciplinary Research Institute, Shanghai University of Traditional Chinese Medicine, Shanghai 201203, China \\ ${ }^{3}$ Chemical Biology, Roche Pharmaceutical Research and Early Development, Roche Innovation Center Shanghai, Shanghai \\ 201203, China \\ ${ }^{4}$ Department of Biological Sciences, Oakland University, Rochester, MI 48309, USA \\ Correspondence to: Yonghua Yang, email: yonghuayang@hotmail.com
}

Keywords: breast cancer, mevastatin, HDAC inhibitor, LBH589, autophagy

Received: November 30, $2016 \quad$ Accepted: January 11, $2017 \quad$ Published: January 27, 2017

\section{ABSTRACT}

Histone deacetylase inhibitors (HDACi) are promising anti-cancer agents, and combining a HDACi with other agents is an attractive therapeutic strategy in solid tumors. We report here that mevastatin increases HDACi LBH589-induced cell death in triple-negative breast cancer (TNBC) cells. Combination treatment inhibited autophagic flux by preventing Vps34/Beclin 1 complex formation and downregulating prenylated Rab7, an active form of the small GTPase necessary for autophagosomeIysosome fusion. This means that co-treatment with mevastatin and LBH589 activated LKB1/AMPK signaling and subsequently inhibited mTOR. Co-treatment also led to cell cycle arrest in $\mathrm{G2} / \mathrm{M}$ phase and induced corresponding expression changes of proteins regulating the cell cycle. Co-treatment also increased apoptosis both in vitro and in vivo, and reduced tumor volumes in xenografted mice. Our results indicate that disruption of autophagosome-lysosome fusion likely underlies mevastatin-LBH589 synergistic anticancer effects. This study confirms the synergistic efficacy of, and demonstrates a potential therapeutic role for mevastatin plus LBH589 in targeting aggressive TNBC, and presents a novel therapeutic strategy for further clinical study. Further screening for novel autophagy modulators could be an efficient approach to enhance HDACi-induced cell death in solid tumors.

\section{INTRODUCTION}

Breast cancer is the second leading cause of cancerrelated deaths among women in the United States, with over 246,660 new diagnoses expected in 2016 and approximately 40,450 deaths [1]. Current clinical therapies include hormone-based agents that directly target estrogen receptor (ER), progesterone receptor (PR), or human epidermal growth factor receptor 2 (HER2) [2, 3]. Triplenegative breast cancer (TNBC) is ER-, PR- and HER2negative, and accounts for approximately $15 \%$ of all breast cancers [3-5]. TNBC is an aggressive subtype that does not respond to ER-, PR-, or HER2-targeted therapies, and TNBC patient prognoses are poor $[3,6]$.
Histone deacetylase inhibitors (HDACi) induce differentiation, senescence, modulation of immune response, altered angiogenesis, cell-cycle arrest, apoptosis, necrosis, and autophagy in a variety of tumor cells [7-9]. Vorinostat and romidepsin were approved by the FDA for T-cell lymphoma treatment in 2006 and 2009, respectively $[10,11]$. Although HDACi are effective as single agents against a subset of hematological tumors, they are less effective in solid tumors [7]. Still, HDACi have shown promising synergistic or additive antitumor effects in combination with other antitumor agents [9, 12-14].

LBH589 is an HDACi shown to suppress TNBC cell growth at nanomolar concentrations $[15,16]$. LBH589 treatment reduced tumor load in vivo and increased overall 
survival in treated mice $[13,17]$. LBH589 combined with other agents may improve treatment efficacy and provide an attractive therapeutic strategy for TNBC. In this study, we show that the combination of mevastatin [18] and LBH589 inhibits TNBC cell proliferation by downregulating the cell cycle regulator, cyclin D1, upregulating P21 activity, and enhancing apoptosis. We show that mevastatin increases autophagososme formation, but decreases autolysosome maturation, potentiating LBH589-induced TNBC cell death. Our results also demonstrate that cellular stress induced by mevastatin plus LBH589 activates LKB1/AMPK to promote TNBC cell death. This activation inhibited mTOR, p70S6K, and cyclin $\mathrm{D} 1$, and induced apoptosis. In addition, treatment reduced Rab7 prenylation, inhibiting autolysosome maturation. Mevastatin plus LBH589 also decreased tumor volume in an in vivo TNBC xenograft tumor model. Thus, our results show that mevastatin plus LBH589 is a potentially efficacious therapeutic strategy for treating TNBC.

\section{RESULTS}

\section{Mevastatin enhances LBH589-induced cell death and autophagy marker expression in human TNBC cells}

We used the LOPAC library (Sigma) of 1280 pharmacologically active compounds to identify suitable LBH589-synergistic partners in TNBC cells. Six active compounds were found to increase LBH589 antiproliferation activity in MDA-MB-231 cells (Figure 1A).

The HMGCR (3-Hydroxy-3-Methylglutaryl-CoA Reductase) inhibitor, mevastatin, which catalyzes the critical and rate limiting step in cholesterol and isoprenoid biosynthesis through the endogenous mevalonate pathway [19], effectively sensitized cells to LBH589 at sublethal concentrations (25 nM) (Supplementary Table 1). We then examined the effects of mevastatin and LBH589 on cell growth using three TNBC cell lines: MDAMB-231, MDA-MB-468 and MDA-MB-453. After 48 $\mathrm{h}$, cell proliferation was measured via CCK8 assay. All cell lines showed dose-dependent responses to mevastatin or LBH589 treatment. All TNBC cell lines treated with LBH589 alone showed similar median inhibitory concentrations $\left(\mathrm{IC}_{50}\right.$ ) (MDA-MB-231: $36.0 \mathrm{nM}, \mathrm{MDA}-$ MB-468: $41.6 \mathrm{nM}$, MDA-MB-453: $27.1 \mathrm{nM}) . \mathrm{IC}_{50}$ values for mevastatin in MDA-MB-468 and MDA-MB-453 cells were above $30 \mu \mathrm{M}$, and were $8.42 \mu \mathrm{M}$ in MDA-MB-231 cells. Simultaneous treatment with mevastatin and LBH589 (25 nM) inhibited cell growth more than single agent treatments. With LBH589, mevastatin $\mathrm{IC}_{50}$ values improved to $0.75 \mu \mathrm{M}$ in MDA-MB-231 cells, $8.10 \mu \mathrm{M}$ in MDA-MB-468 cells, and $17.94 \mu \mathrm{M}$ in MDA-MB-453 cells (Table 1). In MDA-MB-231 cells, the mevastatin $\mathrm{IC}_{50}$ in combination with LBH589 decreased by more than 10fold compared to mevastatin alone.
Statins may prevent various human malignancies, including breast carcinoma [19-21]. Through interference with mevalonate and subsequently geranylgeranyl diphosphate (GGPP) biosynthesis, statins inhibit HMGCR cellular GGPP reduction, activating AMPK, further repressing mTOR activity, and promoting autophagy [22-24]. Therefore, we analyzed whether LBH589 plus mevastatin treatment would affect autophagy in MDAMB-231 and MDA-MB-468 cells. During autophagosome formation, cytosolic LC3-I is cleaved and lipidated to form LC3-II, followed by recruitment to the early phagophore membrane, making LC3-II an excellent autophagosome marker. p62/SQSTM1, normally degraded as part of the autophagy pathway, is a marker for autophagic flux. Similar to our previous LBH589 findings [25], mevastatin alone induced dose dependent LC3-II accumulation and p62/SQSTM1 reduction in both cell lines (Figure 1B-1C). Mevastatin plus LBH589 further increased LC3-II accumulation and decreased p62/SQSTM1 compared to mevastatin alone. Combination treatment induced p62/ SQSTM1 degradation. Together, these results indicate greater autophagy induction with combination treatment.

Anti-proliferation activity was further analyzed by annexin-V and PI staining, followed by flow cytometry to measure apoptosis. Mevastatin or LBH589 caused slight increases in apoptotic MDA-MB-231 and MDA-MB-468 cells compared with controls. Combination treatments increased apoptotic and necrotic cells compared to either mevastatin or LBH589 alone in a mevastatin dosedependent manner. Annexin-V positive MDA-MB-231 and MDA-MB-468 cells increased from $11 \%$ and $12 \%$ to $69 \%$ and $40 \%$, respectively, after $24 \mathrm{~h}$ treatment. These results show that combination treatments dramatically induced MDA-MB-231 and MDA-MB-468 cell death (Figure 1D-1E). To assess caspase involvement in combination treatment-induced cell death, cleavage of PARP, and caspase 8, 3, and 9 was examined. Dosedependent increased cleavage of caspase 8,3 , and 9, and PARP was observed after $24 \mathrm{~h}$ of combined treatment, compared to single agents (Figure $1 \mathrm{~F}-1 \mathrm{G}$ ).

\section{Mevastatin enhances LBH589-induced $\mathrm{G}_{2}-\mathrm{M}$ phase arrest in human TNBC cells}

Statins inhibit cancer cell proliferation by arresting the cell cycle at $\mathrm{G}_{1}-\mathrm{S}$ phase and inducing apoptosis via both mevalonate-dependent pathway and -independent pathways [26]. We performed cell cycle analyses via flow cytometry. Mevastatin treatment in MDA-MB-231 cells increased the percentage of cells in $\mathrm{G}_{1}-\mathrm{G}_{0}$ phase from $58 \%$ to $81 \%$ after $24 \mathrm{~h}$ in a dose-dependent manner, and decreased cells in S phase. Treatment with LBH589 (25 $\mathrm{nmol} / \mathrm{L}$ ) for $24 \mathrm{~h}$ decreased the percentage of $\mathrm{S}$ phase cells from $36 \%$ to $16 \%$, and increased cells in $\mathrm{G}_{1}-\mathrm{G}_{0}$ and $\mathrm{G}_{2}-\mathrm{M}$ phases. Combination treatment in MDA-MB-231 cells increased $\mathrm{G}_{2}-\mathrm{M}$ phase arrest from $5 \%$ to $55 \%$ after $24 \mathrm{~h}$ 
Table 1: IC $_{50}$ of mevastatin on TNBC cell growth with or without LBH589

\begin{tabular}{ccccc}
\hline & Cell lines & $\begin{array}{c}\mathbf{I C}_{\mathbf{5 0}}(\boldsymbol{\mu M M}) \\
\mathbf{M E V}\end{array}$ & $\begin{array}{c}\mathbf{I C}_{\mathbf{5 0}}(\mathbf{n M}) \\
\mathbf{L B H 5 8 9}\end{array}$ & $\begin{array}{c}\mathbf{I C}_{\mathbf{5 0}}(\boldsymbol{\mu M}) \mathbf{M E V} \\
(\mathbf{2 5} \mathbf{~ n M ~ L B H})\end{array}$ \\
\hline \multirow{3}{*}{ Breast Cancer } & MDA-MB-231 & 8.42 & 36.0 & 0.75 \\
& MDA-MB-468 & $>30$ & 41.6 & 8.10 \\
& MDA-MB-453 & $>30$ & 27.1 & 17.94 \\
\hline
\end{tabular}

in a mevastatin dose-dependent manner, and decreased cells in $\mathrm{S}$ and $\mathrm{G}_{1}-\mathrm{G}_{0}$ phases (Figure 2A). A similar cell cycle distribution was observed in MDA-MB-468 cells (Figure 2B): 24-hour treatment with both mevastatin and LBH589 enhanced G2-M phase arrest (from 12\% to $43 \%$ ) and reduced cells in $\mathrm{S}$ and $\mathrm{G}_{1}-\mathrm{G}_{0}$ phases. Cell cycle proteins were analyzed by Western blotting. $24 \mathrm{~h}$ combination treatments in MDA-MB-231 and MDAMB-468 cells downregulated cyclin D1 and survivin, and upregulated p21 in a mevastatin dose-dependent manner (Figure 2C-2D).

\section{Mevastatin enhancement of LBH589-induced cell death is dependent on caspase 8 activity}

To determine whether combined treatment-induced apoptosis was caspase-dependent, we treated cells with the caspase 8 inhibitor z-IETD-fmk (IETD) and the pancaspase inhibitor z-VAD-fmk (z-VAD), and analyzed apoptotic cells via FACS and immunoblotting. z-VAD treatment alone did not affect Annexin-V binding to cells. However, z-VAD addition to mevastatin $(1 \mu \mathrm{M})$ and LBH589 (25 nM)-treated cells increased the viable cell fraction from $36 \%$ to $80 \%$ in MDA-MB-231 cells, and from $53 \%$ to $86 \%$ in MDA-MB- 468 cells (Figure $3 \mathrm{~A}-3 \mathrm{~B}$ ). $z$-VAD rescued the combination treatment in a dosedependent manner. Similar to z-VAD, IETD protects both MDA-MB-231 and MDA-MB-468 cells against mevastatin and LBH589-induced apoptosis (Figure 3A-3B). z-VAD and IETD, but not caspase 9-specific inhibitor LEDH, consistently abrogated cleavage of caspases 8 and 3 , and PARP induced by 24 -h mevastatin $(1 \mu \mathrm{M})$ and LBH589 $(25 \mathrm{nM})$ treatment in both cell lines (Figure $3 \mathrm{C}-3 \mathrm{~F})$. These results indicate that combination treatmentinduced apoptosis is caspase 8-dependent.

\section{Mevastatin plus LBH589 activates the LKB1- AMPK energy-sensing pathway}

We evaluated the effects of combination treatment on intracellular AMPK, AKT, and p42/p44 MAPK (ERK1/2) signaling activation in MDA-MB-231 and MDA-MB-468 cells. 24-h mevastatin and LBH589 treatment increased AMPK $\alpha$ phosphorylation at Thr-172, compared to mevastatin alone, in a dose-dependent manner (Figure 4A-4B). AMPK activation is associated with decreased mTOR and p70S6K activation [27]. Combined treatment decreased mTOR activation, as shown by dosedependent decreased mTOR expression and reduced phosphorylated p70S6K, compared to single agents and controls (Figure 4A-4B). LKB1 is a tumor suppressor that phosphorylates AMPK at Thr-172 [28]. Its activation was investigated by measuring AMPK phosphorylation at Thr-172 and LKB1 Ser-428 phosphorylation. Combination treatment decreased phosphorylation of AKT and ERK, but not MEK, indicating that the combination effect did not activate Ras/Raf signaling (Figure 4C-4D).

For Western blotting analyses, cells were treated with mevastain $(2 \mu \mathrm{M}$ in MDA-MB-231 cells or 16 $\mu \mathrm{M}$ in MDA-MB-468 cells) plus $25 \mathrm{nM}$ of LBH589 for 6, 12, 24 and $36 \mathrm{~h}$ (Figure 4E-4F). Increased AMPK phosphorylation and decreased mTOR were accompanied by reduced downstream p70S6K phosphorylation, decreased cyclin D1, and increased PARP activation in a time-dependent manner.

\section{Mevalonate pathway inhibition and LKB1/ AMPK activation are involved in mevastatin and LBH589-dependent TNBC cell death}

Mevastatin specifically inhibits the rate-limiting enzyme, HMGCR, in the mevalonate pathway. We found that combination treatment-induced cell proliferation inhibition was reversed by addition of mevalonate. $250 \mu \mathrm{M}$ of mevalonate (MVA) impaired mevastatin (MEV) antiproliferation activity in both MDA-MB-231 and MDAMB-468 cells, and recovered cell viability from $73 \%$ and $65 \%$ to $94 \%$ and $91 \%$, respectively (Figure $5 \mathrm{~A}-5 \mathrm{~B}$ ). Moreover, the mevastatin plus LBH589 synergistic effect was abolished by mevalonate addition (Figure. 5A-5B). Cell viability increased from $14 \%$ and $7.8 \%$ to $45 \%$ and $52 \%$, respectively, in high mevastatin dose $(2 \mu \mathrm{M}$ and $16 \mu \mathrm{M}$ )-treated MDA-MB-231 and MDA-MB-468 cells. However, mevalonate did not reverse the effect of LBH589 on cell growth in either cell line, indicating that the mevalonate pathway does not participate in LBH589induced cell death.

In addition to the mevalonate pathway, our results suggested that combination treatment synergy requires AMPK and mTOR signaling. Compound $\mathrm{C}(\mathrm{C}$ in Figures) is an AMPK inhibitor that blocks AMPK metabolic and anti-apoptotic activities [29]. TNBC cells 
were treated with compound C, mevastatin or LBH589 alone or in combination for $48 \mathrm{~h}$. Compound $\mathrm{C}$ alone or with LBH589 or mevastatin had a marginal effect on cell viability. However, compound $\mathrm{C}$ at a dose of $2 \mu \mathrm{M}$ improved proliferation from $31.4 \%$ to $57.9 \%$ and $15.0 \%$ to $57.1 \%$ in MDA-MB-231 cells treated with LBH589 (25 nM) and mevastatin at $1 \mu \mathrm{M}$ and $2 \mu \mathrm{M}$, respectively.
At $1 \mu \mathrm{M}$, compound $\mathrm{C}$ improved MDA-MB-231 cell proliferation from $31.4 \%$ and $15.0 \%$ to $48.1 \%$ and $36.3 \%$ in cells treated with LBH589 (25 nM) and mevastatin at $1 \mu \mathrm{M}$ and $2 \mu \mathrm{M}$ respectively. $2 \mu \mathrm{M}$ compound $\mathrm{C}$ rescued MDA-MB-468 cell viability after treatment with LBH589 $(25 \mathrm{nM})$ and mevastatin at 8 or $16 \mu \mathrm{M}$ from $41.8 \%$ and $26.9 \%$ to $65.9 \%$ and $53.5 \%$, respectively, and $1 \mu \mathrm{M}$
A

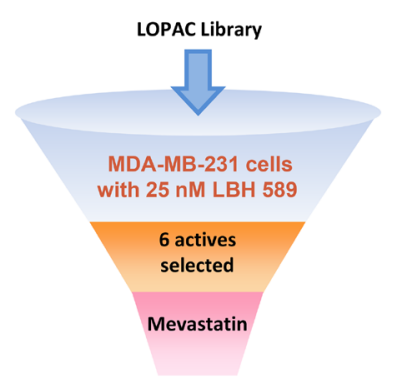

C

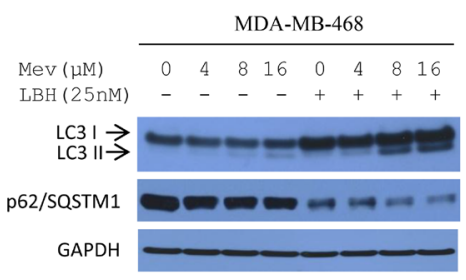

E

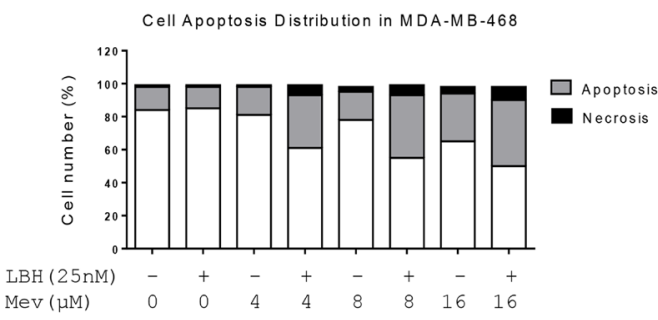

G

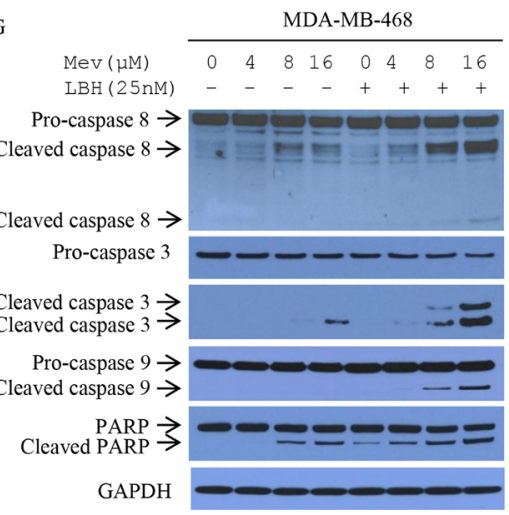

B

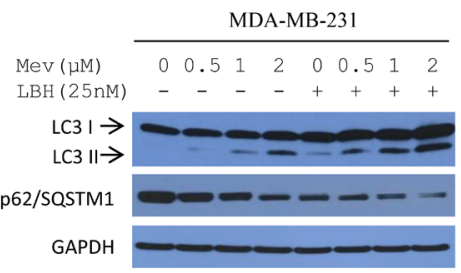

$\mathrm{D}$

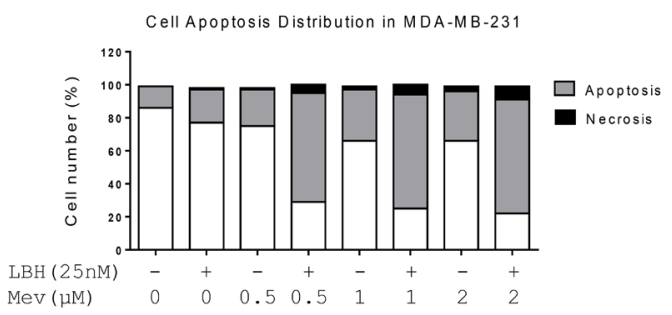

$\mathrm{F}$

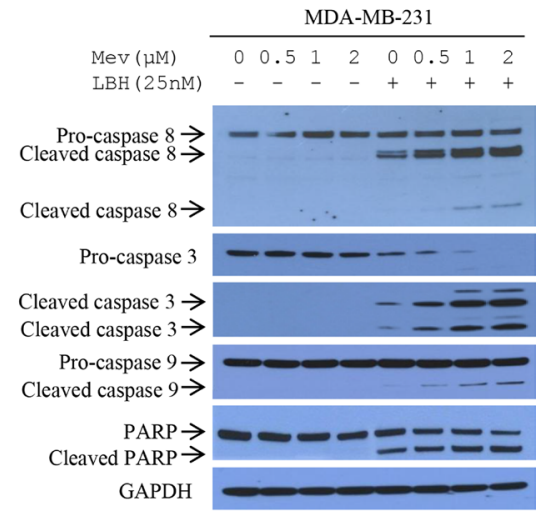

Figure 1: Mevastatin enhances LBH589-induced autophagy and cell death in TNBC cells. Screening for suitable partners acting in synergy with LBH589 in TNBC cells (A) With or without LBH589 (25 nM), endogenous LC3B and p62/SQSTM1 levels were detected by Western blotting in mevastatin-treated MDA-MB-231 $(0,0.5,1,2 \mu \mathrm{M})(\mathbf{B})$ and MDA-MB-468 cells $(0,4,8,16 \mu \mathrm{M})(\mathbf{C})$ for $24 \mathrm{~h}$. Synergistic cell death induction by mevastatin and LBH589 for $24 \mathrm{~h}$ in MDA-MB-231 (D) and MDA-MB-468 cells (E) followed by FACS analysis. Mevastatin enhanced LBH589-induced apoptosis-related proteins dose-dependently in MDA-MB-231 (F) and MDAMB-468 cells $(\mathbf{G})$ as shown by Western blotting. 
compound $\mathrm{C}$ rescued cell viability from $41.8 \%$ and $26.9 \%$ to $59.9 \%$ and $43.2 \%$ (Figure $5 \mathrm{C}-5 \mathrm{D}$ ), respectively.

Western blotting confirmed that combination treatment induced apoptosis through mevalonate pathway blockage and LKB1/AMPK activation. Combined treatment increased AMPK $\alpha$ phosphorylation at Thr-172 compared to single agents and controls (Figure 4A-4B and 5E-5F). Increased AMPK activation inactivated mTOR, as evidenced by diminished mTOR, p-p70S6K, and cyclin D1 levels in both MDA-MB-231 and MDA-MB-468 cells. Combination treatment-induced AMPK, p70S6K, and LKB1 phosphorylation was abolished by $250 \mu \mathrm{M}$ of mevalonate. p70S6K phosphorylation was subsequently restored by $2 \mu \mathrm{M}$ of compound $\mathrm{C}$, which decreased AMPK, but not LKB1, phosphorylation. We also tested whether combination treatment-induced apoptosis could be rescued through the AMPK and mevalonate pathways. Indeed, apoptosis-related protein cleavage was reduced by mevalonate or compound C (Figure 5G-5H). This suggests that both AMPK and mevalonate signaling participate in combination treatment-induced TNBC cell apoptosis.

\section{Mevastatin abrogates LBH589-induced autophagy in TNBC cells via mevalonate pathway blockade}

Consistent with autophagic regulation of apoptosis [30], treatment with LBH589 and mevastatin induced apoptosis in TNBC cells. Mevastatin treatment alone resulted in LC3-II accumulation and p62/SQSTM1 downregulation (Figures 1B-1C and 6A-6B). Combination treatment increased LC3-II and decreased p62/SQSTM1 as compared to either agent alone. This indicates that mevastatin might induce autophagy. However, unlike p62/SQSTM1 downregulation, LBH589mediated NBR1 downregulation was inhibited by combination treatment, which suggests that mevastatin inhibits rather than induces autophagy. To address this, we assessed Vps34 and Beclin 1, essential molecules in autophagic, vesicle formation. LBH589 and mevastatin co-treatment downregulated Vps34 and Beclin 1 (Figure 6A-6B). This effect was rescued by pan-caspase inhibitor, z-VAD. However, z-VAD abrogates caspase 8 activation and enhances LC3-II accumulation. From these results, we could not conclusively demonstrate that mevastatin (with or without LBH589) induced autophagy.

We performed qPCR to determine whether mevastatin or combination treatment-induced p62/SQSTM1 downregulation was due to transcriptional inhibition. Mevastatin suppressed p62/SQSTM1 expression, and this was more pronounced with co-treatment (Figure 6C). Thus, mevastatin inhibits p62/SQSTM1 transcription, but not through autophagy induction.

Combination treatments showed pronounced dosedependent Vps34 and Beclin1 reduction observed after $24 \mathrm{~h}$, compared with single agents in MDA-MB-231 cells (Figure 6D). Mevastatin abolished LBH589-induced Beclin1Vps34 complex formation (Figure 6E). These results,
A

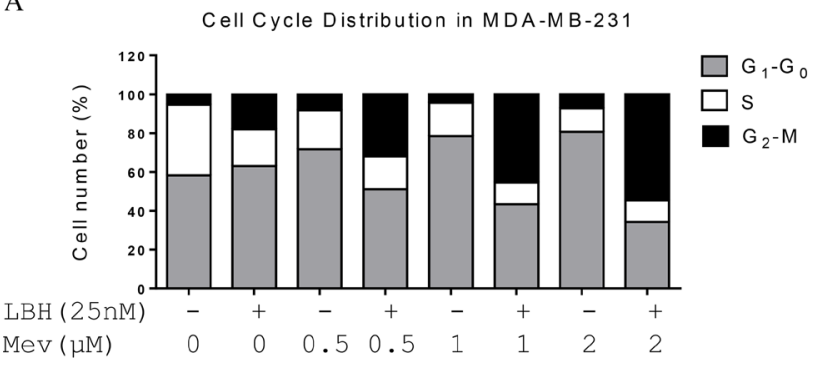

$\mathrm{C}$

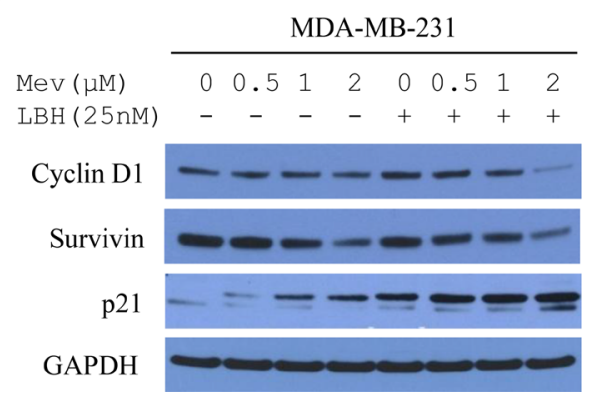

B

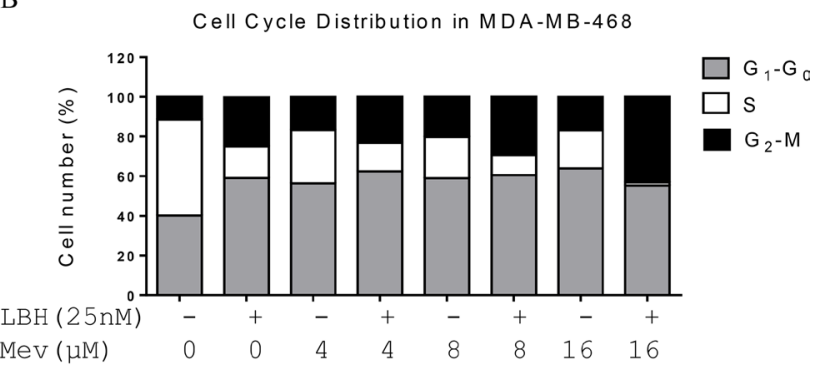

$\mathrm{D}$

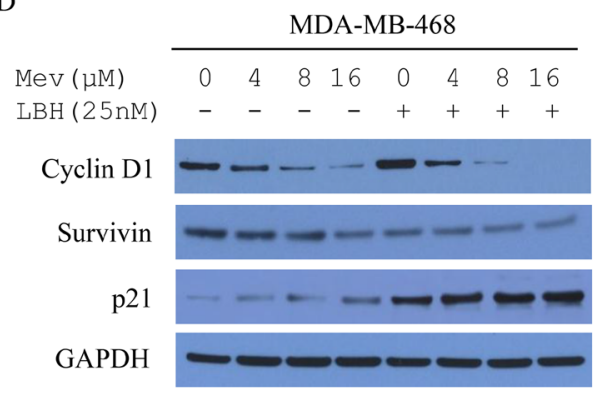

Figure 2: Mevastatin enhances LBH589-induced G2-M arrest in TNBC cells. Cell cycle distribution after treatment with or without LBH589 $(25 \mathrm{nM})$ plus mevastatin $(0,0.5,1,2 \mu \mathrm{M}$ in MDA-MB-231 cells (A); 0, 4, 8, $16 \mu \mathrm{M}$ in MDA-MB-468 cells (B) for $24 \mathrm{~h}$ followed by FACS analysis. Mevastatin regulates cell cycle-related protein expression more efficiently in the presence of LBH589, in a dose-dependent manner. Western blotting of cells treated with or without LBH589 (25 nM) plus mevastatin $(0,0.5,1,2 \mu \mathrm{M}$ in MDAMB-231 cells (C) 0, 4, 8, $16 \mu \mathrm{M}$ in MDA-MB-468 cells (D) for $24 \mathrm{~h}$. 
together with mevastatin-induced NBR1 accumulation, suggest that mevastatin may not only block Beclin1-Vps34 complex assembly to reduce the autophagosome formation, but also may impair autophagosome-lysosome fusion for adaptor protein degradation.

To address whether mevastatin disrupted autophagosome-lysosome fusion, we analyzed co-treated MDA-MB-231 cells stably expressing RFP-GFP-LC3 (tfLC3). tfLC3 contains microtubule-associated protein 1 $\mathrm{LC} 3$ fused with a $\mathrm{pH}$-sensitive tandem fluorescent fusion protein consisting of monomeric red fluorescent protein (mRFP) and enhanced green fluorescent protein (eGFP). GFP, but not mRFP, signals are quenched in acidic environments after autophagosome-lysosome fusion. In initial autophagic vacuoles, tfLC3 emits green and red fluorescence (merged as yellow), whereas in autophagic vacuoles, only red fluorescence is observed. We investigated the effects of the

A

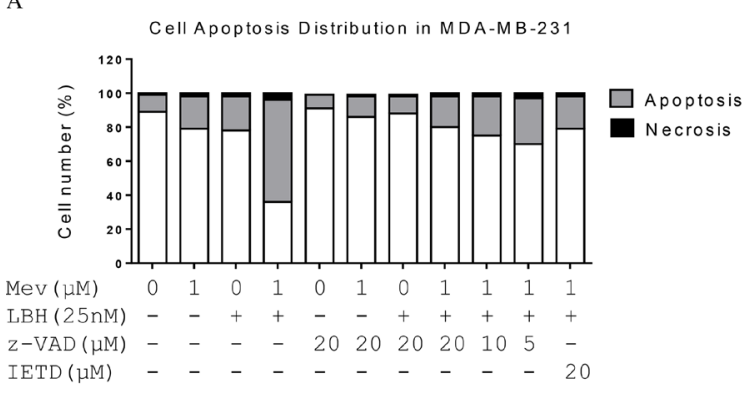

$\mathrm{C}$

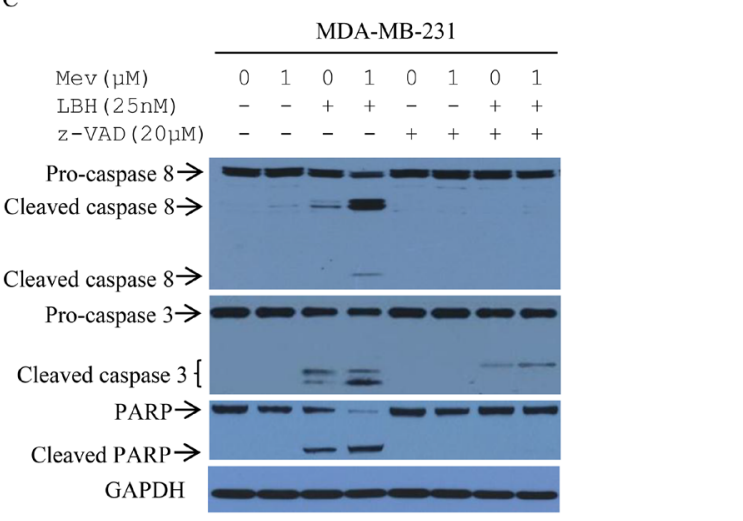

E

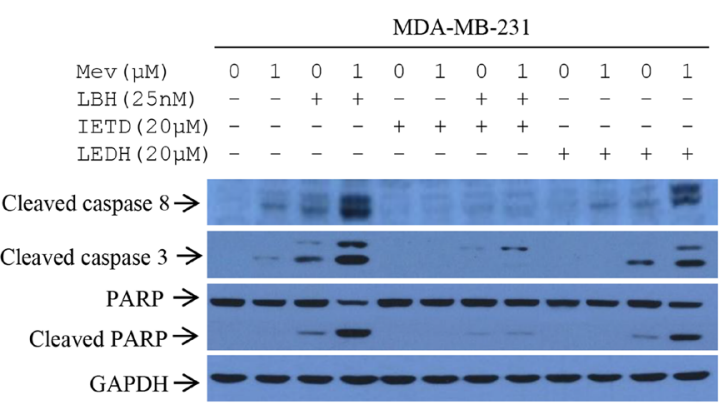

combined treatment on autophagy maturation by comparing colocalized puncta (yellow dots) and mRFP puncta (red dots). Consistent with previous reports [31, 32], LBH589 increased autophagic flux, shown by decreased GFP puncta and increased RFP puncta (Figure 6F). Notably, mevastatin- or LBH589-treated cells differed: both exhibited green and red puncta, but LBH589 treatment produced fewer yellow puncta, indicating that mevastatin induced autophagosome, but not autolysosome maturation. Co-treatment produced more yellow puncta, indicating that combination treatment might block autophagosome-lysosome fusion to inhibit autophagic flux.

Rab7 is a small GTPase critical in autophagosome/ endosome maturation [33, 34], and its activation requires prenylation. To confirm that mevastatin interrupts autophagosome-lysosome fusion mainly via Rab7, cell death was analyzed in Rab7 overexpressing MDA-MB-231 cells treated with LBH589 plus mevastatin. Rab7 overexpressing

B

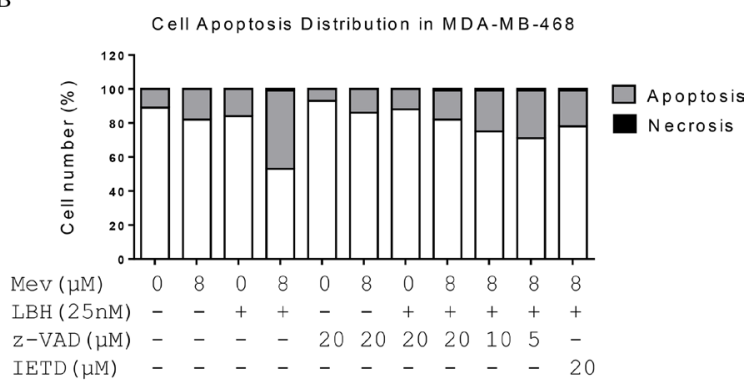

D

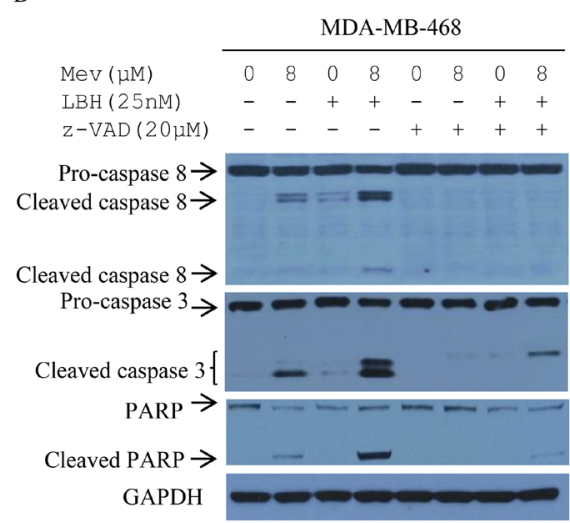

F

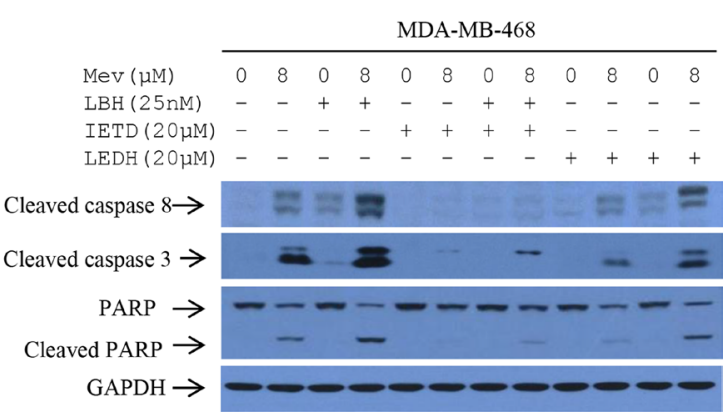

Figure 3: Effect of mevastatin on LBH589-induced cell death. MDA-MB-231 and MDA-MB-468 cells were treated with indicated agents for $24 \mathrm{~h}$. Prior to treatment, $\mathrm{z}$-VAD $(0,5,10,20 \mu \mathrm{M})$ or IETD $(20 \mu \mathrm{M})$ was added for $2 \mathrm{~h}$ to MDA-MB-231 (A) and MDAMB-468 cells (B), and apoptosis was assessed via FACS analysis. Cleaved PARP, and caspases 8 and 3 were analyzed via Western blotting with MDA-MB-231 (C and E) and MDA-MB-468 cells (D and F). 
A

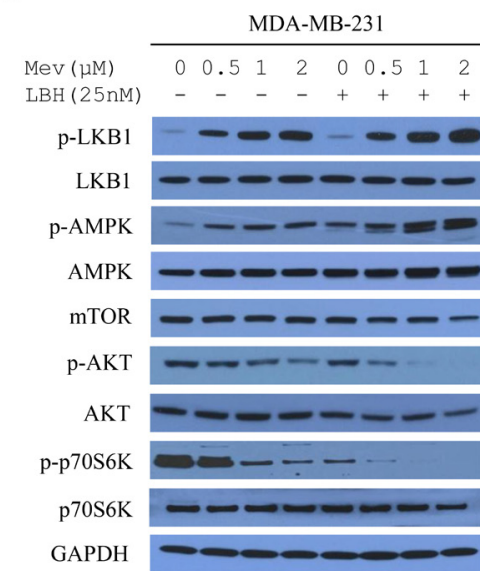

C

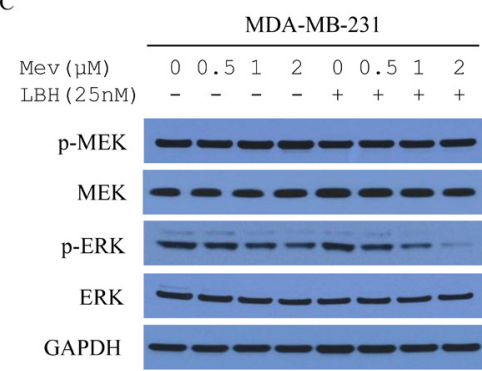

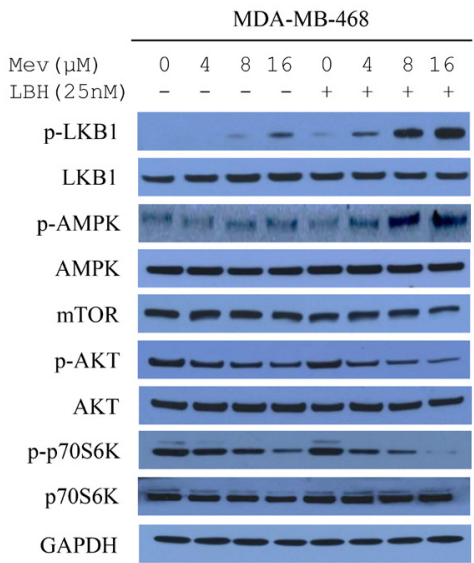

D

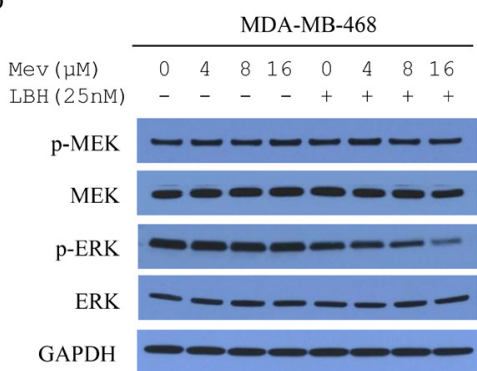

E

MDA-MB-231

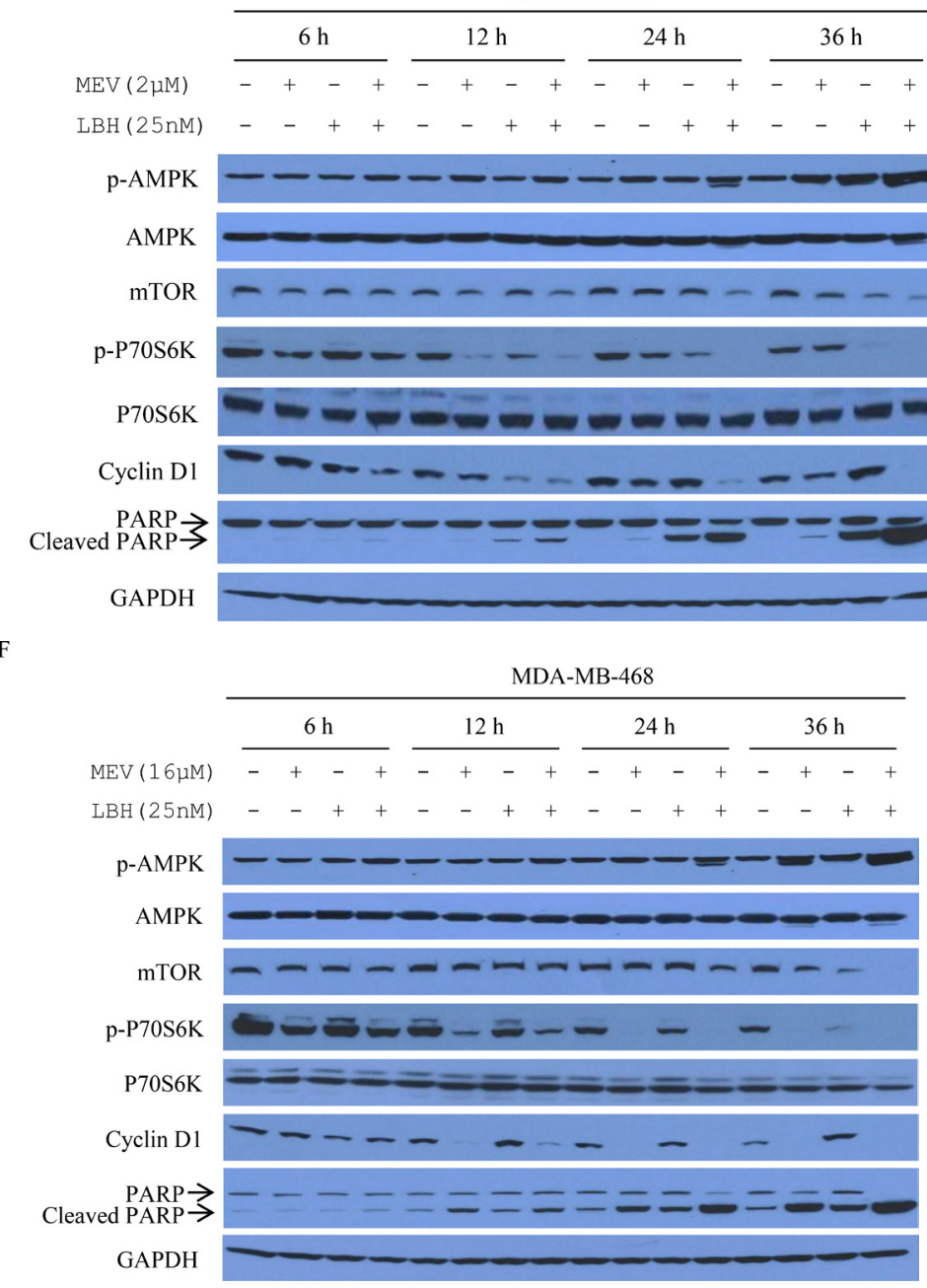


Figure 4: Mevastatin and LBH589 activate AMPK and inhibit mTOR in TNBC cell lines. AMPK involvement in the synergistic effects of mevastatin and LBH589 on MDA-MB-231 (A) and MDA-MB-468 (B) cells for 24 h. Cell lysates were immunoblotted with indicated antibodies. Co-treatment effect on ERK does not depend on Ras in MDA-MB-231 (C) and MDA-MB-468 (D) cells after $24 \mathrm{~h}$. Cell lysates were analyzed by Western blotting for phosphorylated or total MEK, phosphorylated or total ERK, with GAPDH as an internal control. MDA-MB-231 (E) and MDA-MB-468 cells (F) were treated with mevastatin plus LBH589 for 6, 12, 24 and 36 h. Lysates were immunoblotted with the indicated antibodies.
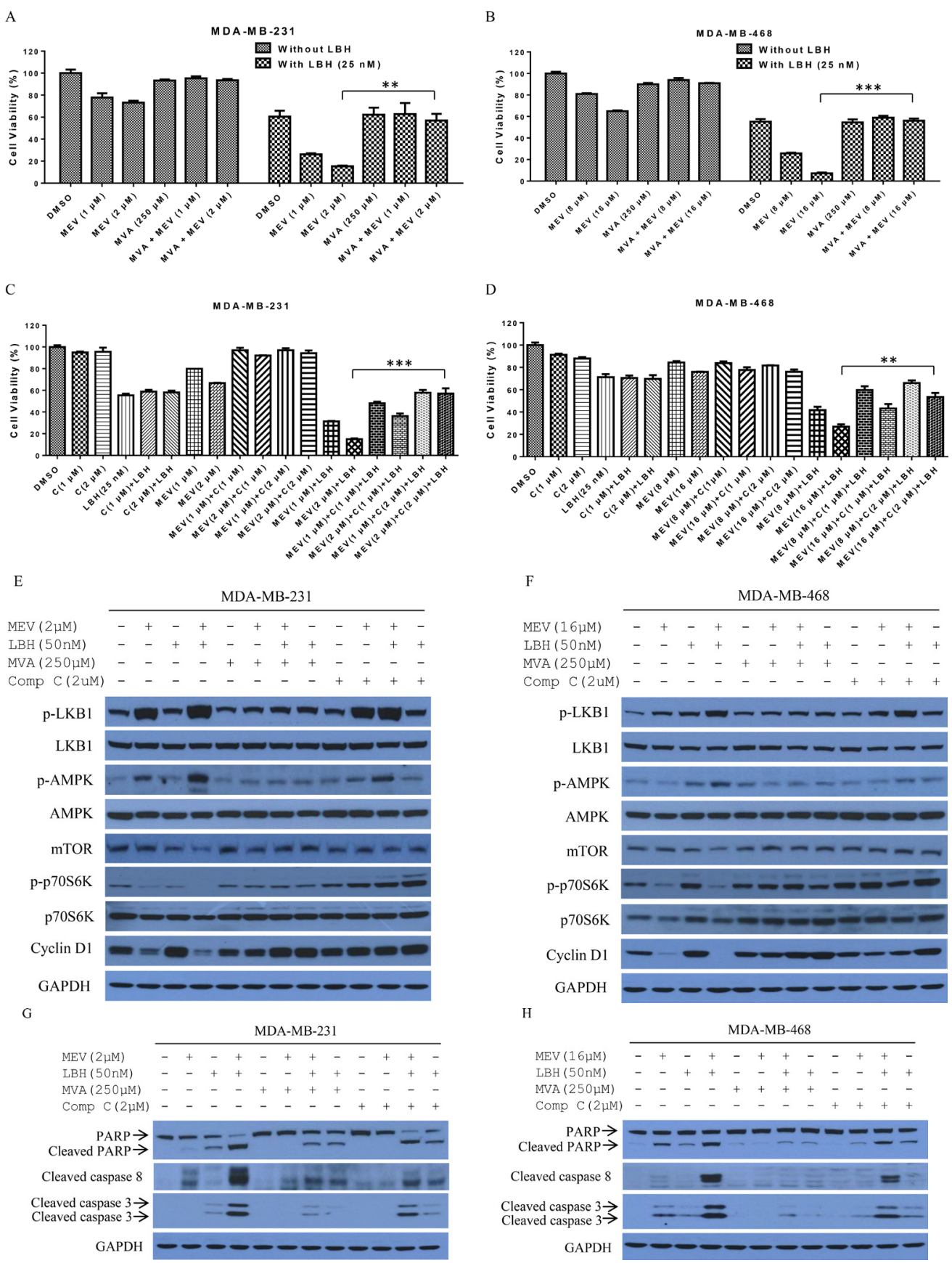

Figure 5: The mevalonate pathway is involved in mevastatin enhancement of LBH-induced TNBC cell death. Mevalonate and compound $\mathrm{C}$ rescued mevastatin-induced proliferation inhibition in MDA-MB-231 and MDA-MB-468 cells. Prior to mevastatin and/ or LBH589 (25 nM) treatment for 48 h, MDA-MB-231 (A) and MDA-MB-468 (B) cells were incubated with or without mevalonate $(250 \mu \mathrm{M})$ for $6 \mathrm{~h}$. Alternatively, prior to treatment, MDA-MB-231 (C) and MDA-MB-468 (D) cells were incubated with or without compound $\mathrm{C}(1,2 \mu \mathrm{M})$ for $2 \mathrm{~h}$. Mevalonate and compound C blocked LKB1/AMPK signaling in MDA-MB-231 (E) and MDA-MB-468 (F) cells treated with indicated agents for $24 \mathrm{~h}$. Cell lysates were immunoblotted with indicated antibodies. Mevalonate and Compound $\mathrm{C}$ reduced cleavage of apoptosis-related proteins in MDA-MB-231 (G) and MDA-MB-468 (H) cells. Cleavage of PARP, and caspases 8 and 3 was analyzed by Western blotting. Data are representative of at least three experiments. Student's $t$-test. ${ }^{* *} P<0.01 ; * * * P<0.001$. 
cells exhibited resistance to combination treatment (Figure $6 \mathrm{G})$. Inefficient fusion with endosomes and/or lysosomes would inhibit autophagosome maturation to amphisomes or autolysosomes, thus inhibiting autophagy. Mevastatin treatment blocked mevalonate signaling, dose-dependently decreasing prenylated Rab7 (Figure 6H). Combination treatment further decreased $\mathrm{Rab} 7$ prenylation in comparison to single agents, suggesting that co-treatment enhanced autophagy blockade. Consistent with confocal microscopy observations, our results indicated that combination treatment blocked autophagosome-lysosome fusion.

To address whether mevalonate signaling was involved in mevastatin abrogation of LBH589-induced autophagy maturation in TNBCs, we treated MDAMB-231 cells with $250 \mu \mathrm{M}$ mevalonate. Immunoblotting showed that mevalonate, but not compound $\mathrm{C}(2 \mu \mathrm{M})$, reversed mevastatin- or combination treatment-induced effects on LC3 II, p62/SQSTM1, NBR1 and prenylated Rab7 (Figure 6I). However, compound $\mathrm{C}$ induced LC3 II accumulation [29]. Taken together, our results demonstrated that mevastatin abrogates LBH589-induced autophagy maturation in TNBCs through blockade of mevalonate signaling.

\section{LBH589 plus mevastatin enhances TNBC death in vivo in MDA-MB-231 xenograft mice}

We established an MDA-MB-231 cell xenograft model in nude mice. Mice were treated with vehicle control, mevastatin (10 mg/kg, orally daily), LBH589 $(0.5 \mathrm{mg} / \mathrm{kg}$, intraperitoneal injection daily), or mevastatin

A

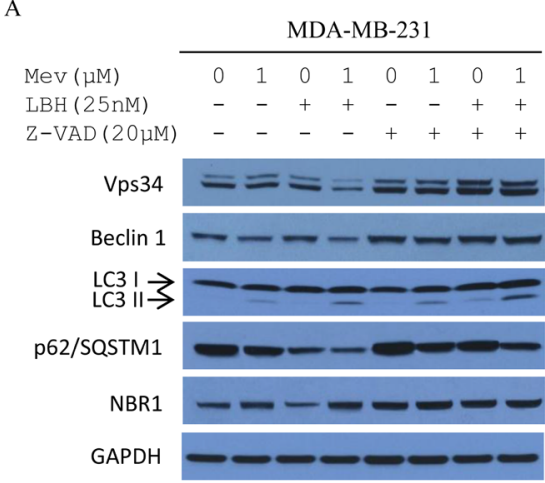

C

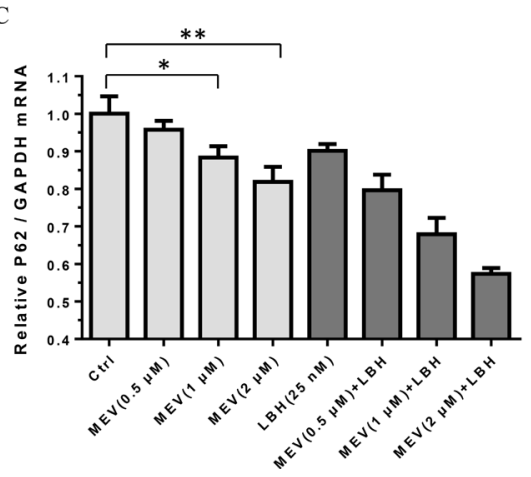

plus LBH589. Treatments began when tumors reached $100 \mathrm{~mm}^{3}$ on average, and tumor growth was measured every five days after treatment initiation. Mevastatin plus LBH589 was more effective in inhibiting tumor growth compared to either mevastatin or LBH589 alone (Figure 7A). Animals treated with mevastatin plus LBH589 exhibited very low tumor growth, with an average final tumor volume of $842 \mathrm{~mm}^{3}$, as compared to controls $\left(3369 \mathrm{~mm}^{3}\right)$, mevastatin alone $\left(1924 \mathrm{~mm}^{3}\right)$, and LBH589 alone $\left(2401 \mathrm{~mm}^{3}\right)$, without obvious toxicity (Figure 7B-7C). Mevastatin stimulated LBH589-induced apoptosis in tumor cells, as demonstrated by TUNEL staining. TUNEL staining showed 2.9\% ( \pm 1.7$)$ apoptosis in control cells, $21.9 \%( \pm 3.2)$ in mevastatin-treated cells, $15.4 \%( \pm 3.6)$ in LBH589-treated cells, and $45.7 \%( \pm 2.1)$ in co-treated cells (Figure 7D-7E).

Finally, we detected Vps34, Beclin 1, LC3 II, p62/ SQSTM1, unprenylated Rab7, prenylated Rab7, caspase 8 , caspase 3 and PARP proteins in xenografted tumor samples. Consistent with our cell culture observations, mevastatin plus LBH589 downregulated Vps34, Beclin1, prenylated Rab7 and p62/SQSTM,1 but elevated LC3 II, unprenylated Rab7, and NBR1 (Figure 7F). Combined treatment enhanced cleavage of caspases 8 and 3, and PARP, compared to single agents and controls. This suggests that mevastatin inhibition of autophagy maturation enhances LBH589-induced apoptosis in TNBCs. Thus, combined treatment leads to tumor cell apoptosis through autophagosome-lysosome fusion blockage and subsequent impaired autophagy maturation. LBH589 plus mevastatin may be an effective novel treatment for aggressive TNBC.

B

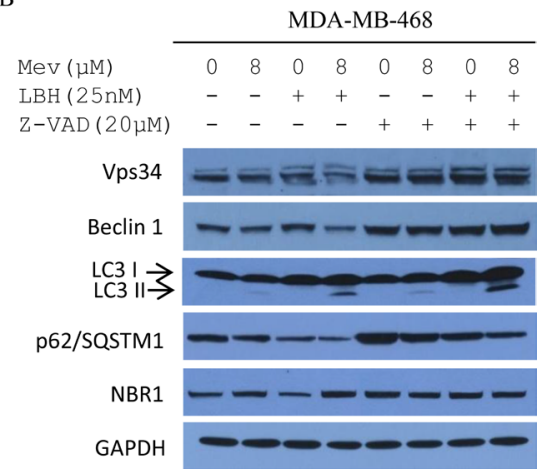

$\mathrm{D}$

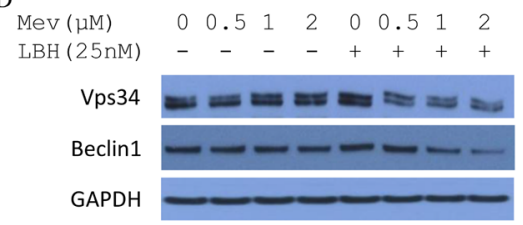




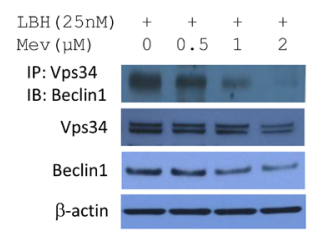

$\mathrm{F}$

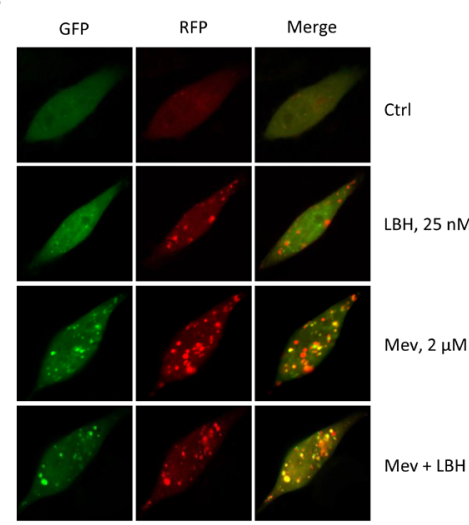

$\mathrm{H}$

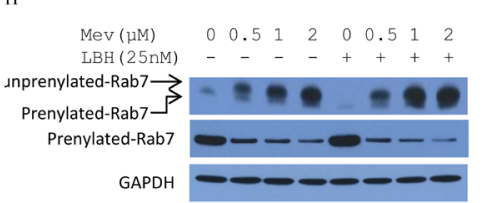

G
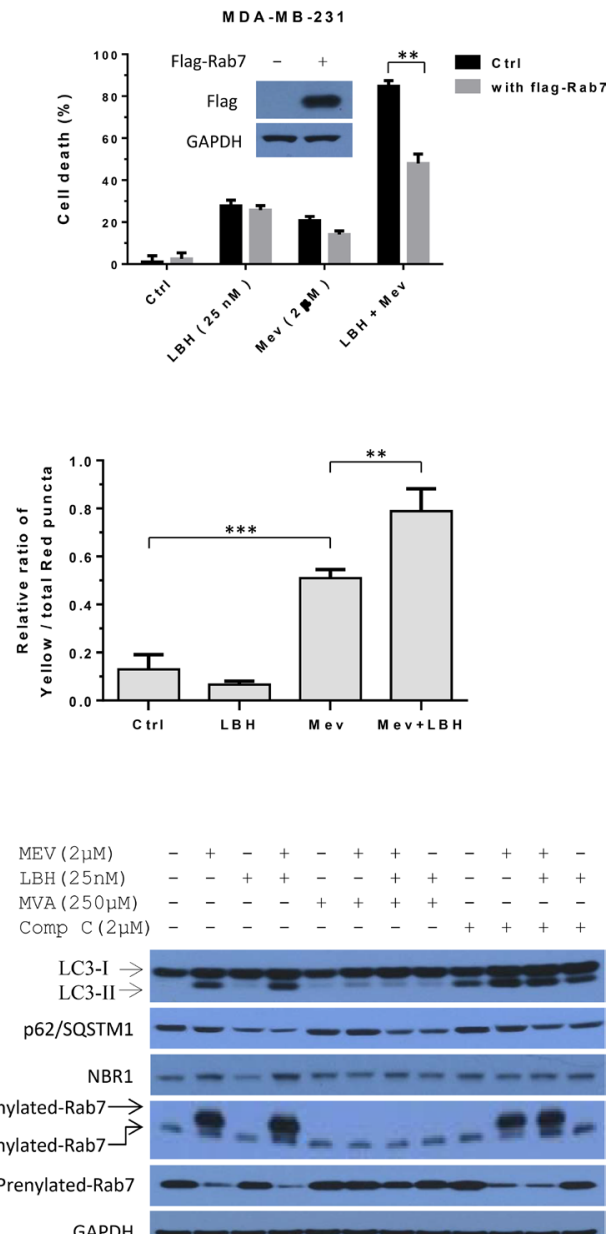

GAPDH

Figure 6: Mevastatin blockade of mevalonate signaling abrogates LBH589-induced autophagy maturation in TNBC cells. Prior to mevastatin plus LBH589 (25 nM) treatment for $24 \mathrm{~h}$, MDA-MB-231 (A) and MDA-MB-468 (B) cells were incubated with or without z-VAD $(20 \mu \mathrm{M})$ for $2 \mathrm{~h}$. Cell lysates were analyzed by Western blotting for Vps34, Beclin 1, LC3B, p62/SQSTM1, and NBR1, with GAPDH as a loading control. Relative p62/SQSTM1 expression (compared with GAPDH) was analyzed by qPCR. Student's $t$-test. ${ }^{*} P<0.05 ;{ }^{* *} P<0.01(\mathbf{C})$. MDA-MB-231 cells were treated with mevastatin $(0,0.5,1,2 \mu \mathrm{M})$ and/or LBH589 (25 nM) for $24 \mathrm{~h}$. Mevastatin reduced LBH589-dependent expression of Vps34 and Beclin 1 in a dose-dependent manner (D). MDA-MB-231 cells were treated with mevastatin $(0,0.5,1,2 \mu \mathrm{M})$ for $24 \mathrm{~h}$ and probed for endogenous Vps34 and Beclin 1. Mevastatin abrogated Vps34-Beclin 1 complex formation dose-dependently in the presence of LBH589 (E). MDA-MB-231 cells were treated with mevastatin $(0,0.5,1,2$ $\mu \mathrm{M})$ plus $25 \mathrm{nM}$ LBH589. Immunoblot analyses were performed on immuoprecipitates as indicated. tfLC3 stable MDA-MB-231 cells were analyzed by confocal microscopy (F). MDA-MB-231_tfLC3 cells were treated with mevastatin $(2 \mu \mathrm{M})$ and/or LBH589 $(25 \mathrm{nM})$ for $24 \mathrm{~h}$. Representative fluorescence images are shown. MDA-MB-231 cells transfected with flag-Rab7 (or pcDNA3.1) were treated with mevastatin $(2 \mu \mathrm{M})$ and/or LBH589 $(25 \mathrm{nM})$ for $48 \mathrm{~h}$, and cell proliferation was measured. Student's $t$-test. ${ }^{* *} P<0.01 ; * * * P<0.001$ (G). Mevastatin with or without LBH589 $(25 \mathrm{nM})$ reduced prenylated Rab7 in a dose-dependent manner (H). MDA-MB-231 cells were treated with mevastatin $(0,0.5,1,2 \mu \mathrm{M})$ and/or LBH589 $(25 \mathrm{nM})$ for 24 hours. Cell lysates were immunoblotted for unprenylated and prenylated Rab7. Prior to treatment with mevastatin $(2 \mu \mathrm{M})$ and LBH589 $(25 \mathrm{nM})$ for $24 \mathrm{~h}$, MDA-MB-231 cells were incubated with or without mevalonate $(250 \mu \mathrm{M})$ for $6 \mathrm{~h}$, or with or without compound C $(2 \mu \mathrm{M})$ for $2 \mathrm{~h}(\mathbf{I})$. Cell lysates were immunoblotted for LC3B, p62/ SQSTM1, and unprenylated and prenylated Rab7, with GAPDH as a loading control.

\section{DISCUSSION}

HDAC dysregulation in cancer cells leads to gene expression changes and regulates cancer cell viability $[12,35]$. Drugs that inhibit HDAC in cancer cells increased survival in mice bearing TNBC cell xenografts $[16,31]$. However, HDACi exhibit only limited effectiveness in low concentrations in solid tumors [7, 12]. A potential solution to this limitation is combination therapy. LBH589 has been evaluated in clinical trials in combination with other drugs $[8,11,15]$. Our study employed an unbiased combinatorial screening, including over 1000 drug combinations, to assess synergistic effects of LBH589 with other drugs for TNBC therapeutic application. 
We found that treatment with LBH589 and mevastatin synergistically reduced MDA-MB-231 and MDA-MB-468 cell proliferation and increased apoptosis (Supplementary Figure 1). Co-treatment decreased tumor growth in in vivo MDA-MB-231 xenografts with minimal toxicity.

Statins reportedly exert anti-tumor and antiproliferation activities in vitro and in vivo, and the clinical utility of these drugs continues to evolve [36]. Statins may exert anti-cancer effects through decreased mevalonate synthesis, and downregulation of products downstream of mevalonate, including dolichol, geranylpyrophosphate and farnesylpyrophosphate. Recent studies indicate important roles for statins in autophagy: autophagy induction through decreased GGPP, and autophagy blockade through decreased prenylated RhoA [22, 37-41].
We investigated the effects of mevastatin alone or in combination with LBH589 on the initial stage of autophagy, autophagic vesicle formation, and the late stage, autolysosome formation. We employed RFPGFP-LC3 (tfLC3) fluorescent analysis to investigate the hypothesis that autophagy blockade is involved in the cellular response to co-treatment. A ratio of yellow/RFPpositive puncta $=1$ indicated autophagy blockage, while a ratio $<1$ indicated abnormally reduced autophagosomelysosome fusion. The decreased yellow/RFP-positive puncta ratio in LBH589-treated cells confirmed autophagy induction and increased autolysosome formation as previously reported [25]. Our results also demonstrated that mevastatin induced autophagy, but this was partially impaired by reduced autophagosome-lysosome fusion, as

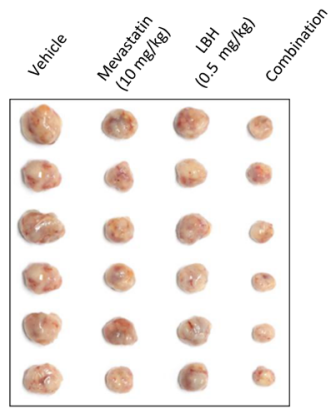

C
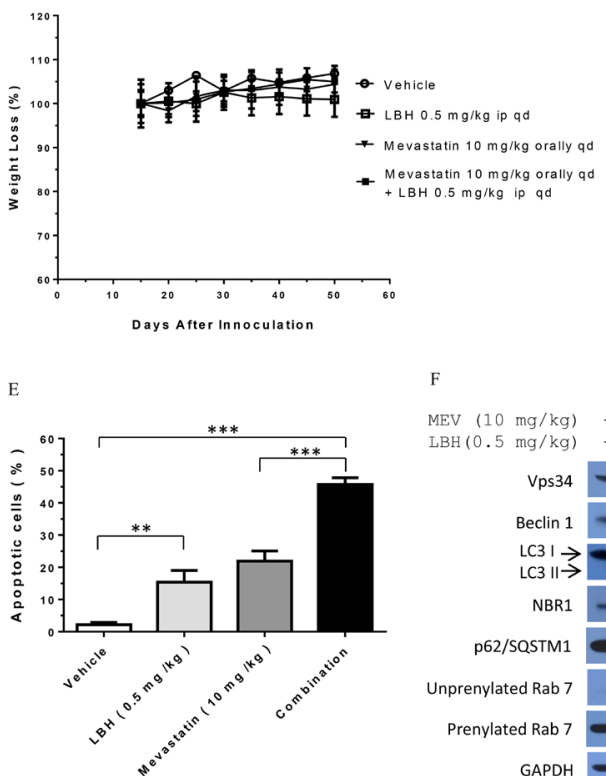

F

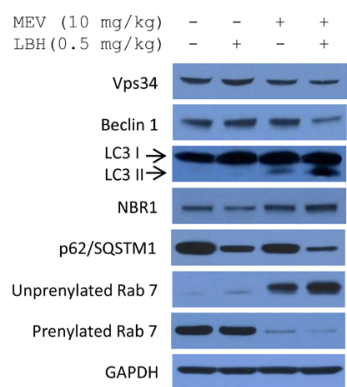

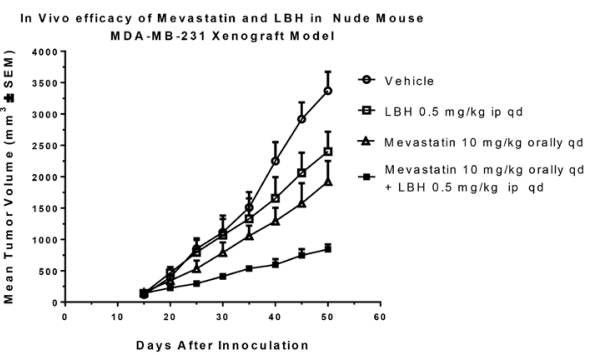

D
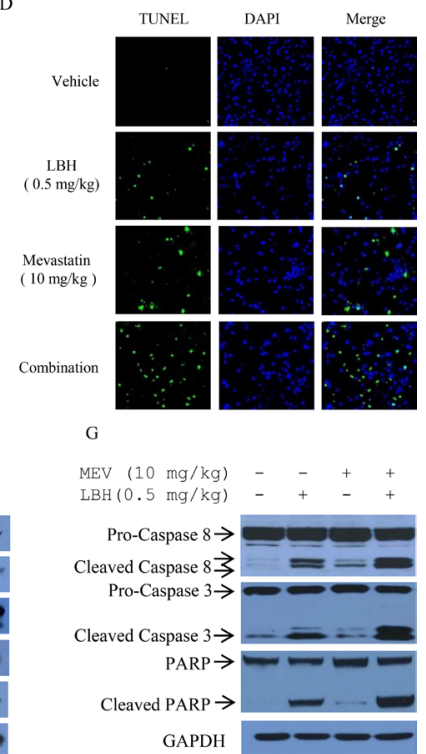

GAPDH

\section{政}

$$
\text { (1) }
$$

Figure 7: Mevastatin plus LBH589 enhances TNBC cell death in vivo in MDA-MB-231 cell xenografted mice. Photographs of subcutaneous tumors resulting from indicated treatments in xenografted nude mice (A). Tumor-bearing mice were treated with vehicle control, mevastatin (10 mg/kg orally daily), LBH589 $(0.5 \mathrm{mg} / \mathrm{kg}$ intraperitoneal injection daily), or combination as indicated (B). Mouse body weight curves following treatments (C). Detection of DNA strand breaks by TUNEL assay in MDA-MB-231 tumors after treatment with vehicle, mevastatin $(10 \mathrm{mg} / \mathrm{Kg}$ orally daily), LBH589 (0.5 mg/kg intraperitoneal injection daily), or combination (D) Green fluorescence indicates TUNEL positive cells. Tumors were stained with DAPI to identify nuclei. Quantitative evaluation of TUNEL positive cells treated with indicated agents $(\mathbf{E})$. Five images were analyzed in each group; bars over columns represent means $\pm \mathrm{SD}$; Student's $t$-test. ${ }^{*} P<0.01 ; * * * P<0.001$. Immunoblotting analysis of xenograft tumor tissues (F and $\left.\mathbf{G}\right)$. 
indicated by yellow/RFP-positive puncta. In co-treated cells, overwhelming RFP-positive/GFP-positive puncta co-localization indicated disruption of autolysosome maturation and subsequent blockade of autophagy flux.

Rab7 is one of the most important effectors in autophagolysosome maturation, promoting microtubule plus-end-directed transport, and facilitating autophagosome-lysosome fusion [34]. Our results showed that mevastatin or mevastatin-LBH589 cotreatment decreased autophagosome-lysosome fusion through downregulation of prenylated Rab7. This could be reversed by mevalonate addition, or partially rescued by the AMPK inhibitor, compound $\mathrm{C}$, indicating that the mevalonate pathway is involved in autophagosomelysosome fusion through Rab7 prenylation. Other studies also suggest that lysosomal positioning proteins, such as TFEB (transcription factor EB) and the AAA-type ATPase SKD1, also impair autophagosome-lysosome fusion $[42,43]$. Thus, other factors may contribute to the co-treatment autophagosome-lysosome fusion inhibitory effect. Vps34 forms a complex with Beclin 1 in phosphoinositide generation, which is essential for autophagic vesicle formation. Our co-IP results revealed Vps34/Beclin 1 complex formation disruption in mevastatin plus LBH589-treated cells. This blockade inhibits autophagy initiation by reducing autophagosome formation, contributing to disruption of autophagy flux.

In summary, autophagy blockade was examined as a potential mechanism underlying the synergistic enhancement of anticancer activity via mevastatin-LBH589 combination treatment (Figure 8). We investigated the effects of mevastatin on autophagy and apoptosis. We showed that mevastatin inhibits autophagic flux by abrogating autophagosome-lysosome fusion, and this activity is enhanced by LBH589. Notably, we demonstrated that mevastatin stimulates LBH589-induced apoptosis in TNBC cells. The combination effect was observed both in vitro and in vivo in a xenograft model. Our results confirm the synergistic efficacy of, and demonstrate a potential therapeutic role for mevastatin plus LBH589 in targeting aggressive TNBC, and offer a new therapeutic strategy for further clinical study. Further screening for novel autophagy modulators could be an efficient approach to enhance HDACi-induced cell death in solid tumors.

\section{MATERIALS AND METHODS}

\section{Cell lines and reagents}

MDA-MB-231, MDA-MB-468 and MDA-MB-453 cells were from the American Type Culture Collection (ATCC). Cells were maintained in RPMI 1640 medium containing $10 \%$ fetal bovine serum (FBS; Gibco, Australia) at $37^{\circ} \mathrm{C}$ in a humidified incubator with $5 \%$ $\mathrm{CO}_{2}$. Cell Counting Kit-8 (CCK8) was purchased from Dojindo Molecular Tech (CK04-20, Kumamoto, Japan). $\mathrm{X}$-tremeGENE HP DNA Transfection Reagent and In Situ Cell Death Detecting Kit were purchased from Roche diagnostic (Indianapolis, USA). LBH589 was purchased from Selleck Chem (Houston, USA). pLentiC-Myc-DDK-RAB7 (RC201776L1) was purchased from Origene (Rockville, USA). The cDNA encoding mRFPGFP-LC3 (tfLC3) was excised from ptfLC3 (Addgene) and sub-cloned into the lentiviral vector, $\mathrm{pCDH1-MCS1-}$ EF1-puro (System Bioscience). Z-VAD-FMK (FMK001), Z-LEDH-FMK and Z-IETD-FMK were purchased from R\&D Systems (Shanghai, China). Mevastatin (M2537), Mevalonate (M4667), compound C (P5499), ellipticine (E3380), monoamine oxidase (MAO) inhibitor (Q3251), dequalinium dichloride (D3768), tyrphostin A9 (T182) and DEDA (D8008) were purchased from Sigma (Shanghai, China).

\section{Cellular proliferation assay (CCK8 assay)}

Cells were plated in triplicate and incubated with increasing compound concentrations for $48 \mathrm{~h}$. Proliferation was then measured using a WST-8 (2-(2-methoxy-4-

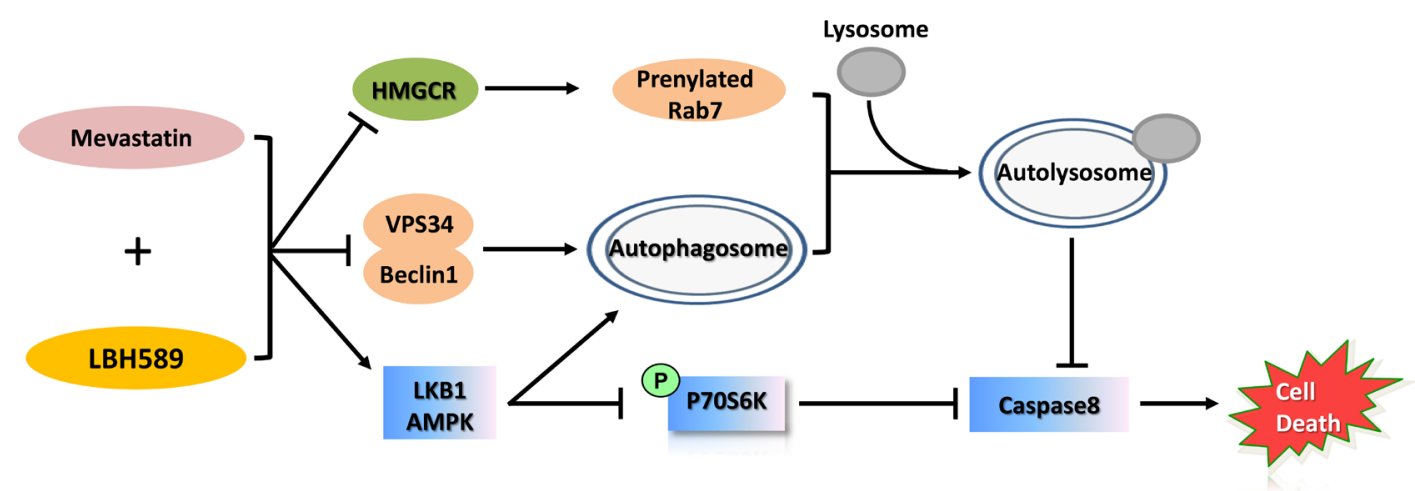

Figure 8: Schematic diagram of the antitumor activities of mevastatin and LBH589 in TNBC. This schematic diagram shows that mevastatin increased HDACi LBH589-induced cell death in TNBC cells. Combination treatment inhibited autophagic flux by preventing Vps34/Beclin 1 complex formation and the maturation of autophagosome-lysosome via down regulated prenylated Rab7 as well as the activity of P70S6K by activated LKB1/AMPK signaling, resulting in Caspase8 dependent cell death. 
nitrophenyl)-3-(4-nitrophenyl)-5- (2, 4-disulfophenyl)$2 \mathrm{H}$-tetrazolium)-based viability assay (CCK8, Dojindo Molecular Tech). Medium was replaced with fresh WST8medium (1:10). Cells were incubated $1-2 \mathrm{~h}$, and absorbance at $450 \mathrm{~nm}$ was measured using an Envision 2104 microplate reader (PerkinElmer). Compound inhibition rate (IR) was determined using the following formula: IR $(\%)=$ $\left(\mathrm{OD}_{\text {Control }}-\mathrm{OD}_{\text {Mevastatin }}\right) / \mathrm{OD}_{\text {Control }} * 100 \%$. For mevastatin and LBH589 combination treatment, IR $(\%)=\left(\mathrm{OD}_{\mathrm{Control}^{-}}\right.$ $\left.\mathrm{OD}_{\text {Mevastatin \& LBH589 }}\right) /\left(\mathrm{OD}_{\text {control }}-\mathrm{OD}_{\text {LBH589 }}\right) * 100 \%$. The curve of IR against tested compound concentrations was determined with SoftMax Pro software, which was also used to find concentrations corresponding to $50 \% \mathrm{IR}\left(\mathrm{IC}_{50}\right)$ on the curve.

\section{Screening of hits in combination with LBH589}

MDA-MB-231 cells was seeded in 384 well plates at a density of 12,000 cells per well. Cellular proliferation assay (CCK8 assay) was used for measurement of the cytotoxic effect. An integrated Agilent Biocel 900 System containing direct drive robot (DDR), Liconic $\mathrm{CO}_{2}$ incubator, Thermo combi dispenser, liquid handler (Bravo), and a multipurpose reader (Envision, PerkinElmer) enabled high-throughput readout of cell survival after compounds treatment. The substances used in combination with LBH589 were 1,280 pharmacologically active compounds from sigma (LOPAC $\left.{ }^{\circledR} 1280\right)$. Cells treated with compounds $(10 \mu \mathrm{M})$ and/or LBH589 (25 nM) for $48 \mathrm{~h}$ followed by cell proliferation detection.

\section{Cell cycle analysis}

MDA-MB-231 and MDA-MB-468 cells were seeded in a 6 -well plate at $5.0 \times 10^{5}$ cells/well and treated with mevastatin and LBH589. $24 \mathrm{~h}$ later, cells were harvested with trypsin and fixed with $\mathrm{EtOH}$ at $4{ }^{\circ} \mathrm{C}$ for $3 \mathrm{~h}$. Cells were then centrifuged at 1,500 $\mathrm{g}$ for $5 \mathrm{~min}$ and washed with PBS. Cells were resuspended in RNase/PBS solution $(20 \mu \mathrm{g} / \mathrm{ml})$, incubated at $37^{\circ} \mathrm{C}$ for $15 \mathrm{~min}$, added to propidium iodide (PI)/PBS solution $(20 \mu \mathrm{g} / \mathrm{ml})$, and incubated at RT for $30 \mathrm{~min}$. Stained cells were analyzed via a FACSCalibur (Becton Dickinson, New Jersey, USA) and data were analyzed using CellQuest Pro software (Becton Dickinson).

\section{Apoptosis analysis}

MDA-MB-231 and MDA-MB-468 cells were seeded in a 6 -well plate at $5.0 \times 10^{5}$ cells/well and treated with mevastatin and LBH589. $24 \mathrm{~h}$ later, cells were harvested with trypsin and washed once with pre-cooled PBS. Cells were re-suspended in $500 \mu 11 \times$ binding buffer, and $5 \mu \mathrm{L}$ PI and $5 \mu \mathrm{l}$ annexin-V (Becton Dickinson) were added. After incubation in the dark (room temperature) for $10 \mathrm{~min}$, stained cells were analyzed via a FACSCalibur instrument and CellQuest Pro software.

\section{Determination of combination effect}

MDA-MB-231 and MDA-MB-468 cells were plated in $96-$ well microplates at $2 \times 10^{4}$ cells/well. Cells were treated for $48 \mathrm{~h}$ with mevastatin from 30 to $0.47 \mu \mathrm{M}$ with 2-fold dilutions, LBH-589 from 150 to $2.3 \mathrm{nM}$ with 2-fold dilutions, or their corresponding combinations. Synergism after drug treatments was quantitated using the combination index (CI) method in ED50, ED75 and ED90 by Calcusyn software. The Chou and Talalay method was used to calculate combination index (CI). CI $>1,=1$, or $<1$ indicates antagonism, additive effect, or synergy, respectively. Each CI ratio is the mean value derived from at least three independent experiments.

\section{Western blotting analysis}

MDA-MB-231 and MDA-MB-468 cells were seeded in a $60 \mathrm{~mm}$ dish and treated with mevastatin and LBH589. $24 \mathrm{~h}$ later, cells were harvested and lysed by RIPA buffer $(150 \mathrm{mM} \mathrm{NaCl}, 1 \% \mathrm{NP} 40,0.25 \%$ deoxycholate and $10 \mathrm{mM}$ Hepes, $\mathrm{pH}$ 7.4) containing a protease inhibitor cocktail (Roche Molecular Biochemicals). Protein concentrations were quantified using the bicinchoninic acid (BCA) method (Pierce). $30 \mu \mathrm{g}$ protein per sample was boiled in loading buffer and electrophoresed in $8-12 \%$ gradient SDS polyacrylamide gels (Invitrogen) and transferred onto nitrocellulose membranes. Membranes were probed with antibodies (AKT1 \#2967, p-AKT1 \#4060, AMPKa \#2603, p-AMPKa \#2535, Beclin1 \#3495, Caspase3 \#9662, Cleaved Caspase3 \#9661, Caspase8 \#9746, Caspase9 \#9502, Cleaved Caspase9 \#9501, Cyclin D1 \#2926, p-Cyclin D1 \#3300, GAPDH \#3683, MEK1/2 \#4694, p-MEK1/2 \#9154, mTOR \#2972, p-mTOR \#2971, p44/42 MAPK (Erk1/2) \#9107, p-p44/42 MAPK (Erk1/2) \#4370, P70 S6 Kinase \#9202, p-P70 S6 Kinase \#9205, LKB1 \#3047, p-LKB1 \#3482, NBR1 \#9891, PARP \#9542, Rab7 \#2094 and Survivin \#2808 were purchased from Cell Signaling Technology; p21 and p53 were purchased from R\&D; VPS34 \#Z-R015 was purchased from Echelon Bioscience; LC3B and $\beta$-actin were purchased from Sigma; and p62/SQSTM1 was purchased from MBL), followed by a peroxidase-conjugated immunoglobulin. Western blots were visualized with an enhanced chemiluminescence (ECL) Plus kit (Pierce, ThermoFisher).

\section{Separation of prenylated and unprenylated proteins}

Triton X-114 fractionation was used to separate prenylated and unprenylated Rab7 in whole cell lysates from cultures treated with mevastatin and LBH589. Triton X-114 enables separation of hydrophilic proteins from amphiphilic proteins; therefore, lipophilic, prenylated Rab7 partitions into the detergent-rich phase, whereas unprenylated Rab7 remains in the aqueous phase. 
MDA-MB-231 cells were harvested following mevastatin and LBH589 treatment by scraping into medium and pelleting via centrifugation at $1500 \times \mathrm{g}$. Cell pellets were washed once in PBS before being lysed in Triton X-114 buffer (20 mM Tris, $150 \mathrm{mM} \mathrm{NaCl}$, pH 7.5, $1 \%$ Triton X-114, protease inhibitor cocktail) for 15-20 $\min$ at $4^{\circ} \mathrm{C}$. Lysates were cleared by centrifugation at $13,000 \times \mathrm{g}$ for $15 \mathrm{~min}$ at $4^{\circ} \mathrm{C}$, and then incubated at $37^{\circ} \mathrm{C}$ for $10 \mathrm{~min}$. Following centrifugation at $13,000 \times \mathrm{g}$ for 2 min at room temperature, the top aqueous clear layer was transferred to a new microfuge tube. Buffer without Triton X-114 (20 mM Tris, $150 \mathrm{mM} \mathrm{NaCl,} \mathrm{pH} \mathrm{7.5,} \mathrm{protease}$ inhibitor cocktail) was added to the bottom detergent-rich layer to equalize the volume with the aqueous phase, and protein was electrophoresed on $12 \%$ polyacrylamide gels. Gels were analyzed by Western blotting for Rab7 and GAPDH.

\section{Quantitative real-time PCR (qPCR) analysis}

MDA-MB-231 cells were harvested following mevastatin and LBH589 treatment. RNA was isolated with RNeasy Plus Mini kits (QIAGEN) according to the supplier's protocol. Total RNAs (500 ng) was reversetranscribed in $25 \mu$ l using TaqMan ${ }^{\circledR}$ Reverse Transcription Reagents (Applied Biosystems, Inc.).

Each qPCR reaction was performed with $2.5 \mu \mathrm{L}$

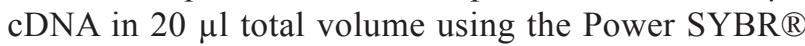
Green PCR Master Mix. Reaction conditions were as follows: initial denaturation at $95^{\circ} \mathrm{C}$ for $10 \mathrm{~min}$ followed by 40 cycles at $95^{\circ} \mathrm{C}$ for $10 \mathrm{~s}, 60^{\circ} \mathrm{C}$ for $10 \mathrm{~s}$ and extension at $72^{\circ} \mathrm{C}$ for $15 \mathrm{~s}$, then $10 \mathrm{~min}$ at $72^{\circ} \mathrm{C}$, and $55^{\circ} \mathrm{C}$ rise to $95^{\circ} \mathrm{C}$ for melt curves. Primers used included: p62/SQSTM1 (5>-GCA CCC CAA TGT GAT CTG C-3〉, 5>-CGC TAC ACA AGT CGT AGT CTG G-3〉) and GAPDH (5>-GGA GCG AGA TCC CTC CAA AAT-3», 5»-GGC TGT TGT CAT ACT TCT CAT GG-3〉). All samples were analyzed in triplicate, and reactions were performed using a LightCycler 480 II PCR machine (Roche Diagnostic, Inc.). PCR product amounts were normalized first to GAPDH, and then to levels in control cells as 1 .

\section{Stable MDA-MB-231_tfLC3 cells, and confocal microscopy}

Briefly, cDNA encoding mRFP-GFP-LC3 (tfLC3) was excised from ptfLC3 (Addgene, 21074) and subcloned into the lentiviral vector, pCDH1-MCS1-EF1puro (System Bioscience, CD510A-1). Then, the recombinant lentivirus encoding tfLC3 was produced to transduce MDA-MB-231 cells, and $5 \mu \mathrm{g} / \mathrm{ml}$ of puromycin (Invitrogen) was used to select for stable tfLC3-expressing cells, referred to here as MDA-MB-231_tfLC3 cells. Images were acquired with an LSM710 confocal microscope with a $63 \times$ objective (Carl Zeiss).

\section{Immunoprecipitation analyses}

After culturing plates for $24 \mathrm{~h}, 2 \times 10^{6}$ cells in 100 $\mathrm{mm}$ dishes were treated with mevastatin and LBH589. Cell lysates prepared in IP lysis buffer (1\% Triton $\mathrm{X}-100,150 \mathrm{mM} \mathrm{NaCl}, 10 \%$ glycerol, $30 \mathrm{mM}$ Tris- $\mathrm{HCl}$, $\mathrm{pH}$ 7.5) containing protease inhibitors were pre-cleaned by incubation with anti-rabbit IgG-conjugated agarose beads (Sigma, A2909) for $2 \mathrm{~h}$ at $4^{\circ} \mathrm{C}$, and subjected to immunoprecipitation with anti-VPS34 monoclonal antibodies (Echelon Bioscience, Z-R015). Resulting immune-complexes were washed three times with the lysis buffer and subjected to immunoblotting analyses.

\section{Transient transfection assay}

$10 \times 10^{5}$ MDA-MB-231 cells were seeded in 6-well plates. $24 \mathrm{~h}$ later, $2 \mu \mathrm{g}$ of flag-Rab7 (or pcDNA3.1) was transfected into cells using $6 \mu \mathrm{l}$ of X-tremeGENE HP DNA transfection reagent (Sigma-Aldrich). $6 \mathrm{~h}$ after transfection, cells were re-suspended and seeded in 96 well plates at $2 \times 10^{4}$ cells/well. After $16 \mathrm{~h}$ incubation, cells were treated with mevastatin $(2 \mu \mathrm{M})$ and/or LBH589 (25 $\mathrm{nM})$ for $48 \mathrm{~h}$ followed by cell proliferation detection. Cell death percentage $(\%)=\left(\mathrm{OD}_{\text {Control }}-\mathrm{OD}_{\text {treatment }}\right) /\left(\mathrm{OD}_{\text {Control }^{-}}\right.$ $\left.\mathrm{OD}_{\text {blank }}\right) * 100 \%$.

\section{In vivo drug activity}

MDA-MB-231 $\left(1 \times 10^{7}\right.$ cells in $0.2 \mathrm{ml}$ PBS $)$ were injected into the left armpits of 5-6-week old BALB/c $\mathrm{nu} / \mathrm{nu}$ athymic female mice (Sino-British SIPPR/BK Lab. Animal Ltd, China). Tumor volume $\left(\mathrm{mm}^{3}\right)$ was measured with calipers and calculated as $(\mathrm{W} 2 \times \mathrm{L}) / 2$, where $\mathrm{W}$ is width and $\mathrm{L}$ is length. When tumor volume reached $\sim 100 \mathrm{~mm}^{3}$, mice were randomized into four groups (five mice per group) including control, mevasatin $(10 \mathrm{mg} / \mathrm{kg}$ orally daily), LBH589 $(0.5 \mathrm{mg} / \mathrm{kg}$ intraperitoneal injection (ip) daily) and mevastatin (10 mg/kg orally daily) plus LBH589 $(0.5 \mathrm{mg} / \mathrm{kg}$ ip daily) groups, and treatments were initialized. Sterilized drinking water containing PBS was supplied to control mice. Tumor volumes were recorded every five days. Animals were sacrificed 35 days postinduction. Tumor tissues were collected and subjected to Western blotting analyses for relative protein expression, and TUNEL assay.

\section{TUNEL assay}

TUNEL (terminal deoxynucleotidyltransferasemediated dUTP nick end labeling) assay was carried out using "In situ cell death detection kit, fluorescein" (Roche, USA) according to the manufacturer's instructions. Slides were rinsed twice with PBS. After drying areas around the sample, $50 \mu 1$ TUNEL reaction mixture was added. After rinsing three times with PBS, samples with fluorescein 
labels incorporated in nucleotide polymers were detected by fluorescence microscopy. Nuclei were counted by counter staining with 4,6-diamidino-2-phenylindole (DAPI) at excitation wavelength, $350 \mathrm{~nm}$.

\section{Statistical analysis}

All results were expressed as means $\pm \mathrm{SD}$ of three independent experiments. Statistical analyses were performed using Student's two-tailed $t$-test by Graphpad Prism 6.07 software. $P<0.05$ was considered significant.

\section{ACKNOWLEDGMENTS AND FUNDING}

This work was supported by grants from the National Natural Science Foundation of China (No. 81272391 and 81572721 to Y.Y.).

\section{CONFLICTS OF INTEREST}

The authors declare no conflicts of interest.

\section{REFERENCES}

1. Siegel RL, Miller KD, Jemal A. Cancer statistics, 2016. CA Cancer J Clin. 2016; 66:7-30.

2. Fernandez Y, Cueva J, Palomo AG, Ramos M, de Juan A, Calvo L, Garcia-Mata J, Garcia-Teijido P, Pelaez I, GarciaEstevez L. Novel therapeutic approaches to the treatment of metastatic breast cancer. Cancer Treat Rev. 2010; 36:33-42.

3. Chacon RD, Costanzo MV. Triple-negative breast cancer. Breast Cancer Res. 2010; 12:S3.

4. Elias AD. Triple-negative breast cancer: a short review. Am J Clin Oncol. 2010; 33:637-645.

5. Hudis CA, Gianni L. Triple-negative breast cancer: an unmet medical need. Oncologist. 2011; 16:1-11.

6. Oakman C, Viale G, Di Leo A. Management of triple negative breast cancer. Breast. 2010; 19:312-321.

7. Zhang J, Zhong Q. Histone deacetylase inhibitors and cell death. Cell Mol Life Sci. 2014; 71:3885-3901.

8. Slingerland M, Guchelaar HJ, Gelderblom H. Histone deacetylase inhibitors: an overview of the clinical studies in solid tumors. Anticancer Drugs. 2014; 25:140-149.

9. Frew AJ, Johnstone RW, Bolden JE. Enhancing the apoptotic and therapeutic effects of HDAC inhibitors. Cancer Lett. 2009; 280:125-133.

10. Mann BS, Johnson JR, Cohen MH, Justice R, Pazdur R. FDA approval summary: vorinostat for treatment of advanced primary cutaneous T-cell lymphoma. Oncologist. 2007; 12:1247-1252.

11. Piekarz RL, Frye R, Turner M, Wright JJ, Allen SL, Kirschbaum MH, Zain J, Prince HM, Leonard JP, Geskin LJ, Reeder C, Joske D, Figg WD, et al. Phase II multi-institutional trial of the histone deacetylase inhibitor romidepsin as monotherapy for patients with cutaneous T-cell lymphoma. J Clin Oncol. 2009; 27:5410-5417.

12. Gryder BE, Sodji QH, Oyelere AK. Targeted cancer therapy: giving histone deacetylase inhibitors all they need to succeed. Future Med Chem. 2012; 4:505-524.

13. Ocio EM, Vilanova D, Atadja P, Maiso P, Crusoe E, Fernandez-Lazaro D, Garayoa M, San-Segundo L, Hernandez-Iglesias T, de Alava E, Shao W, Yao YM, Pandiella A, et al. In vitro and in vivo rationale for the triple combination of panobinostat (LBH589) and dexamethasone with either bortezomib or lenalidomide in multiple myeloma. Haematologica. 2010; 95:794-803.

14. Lemaire $M$, Fristedt C, Agarwal P, Menu E, Van Valckenborgh E, De Bruyne E, Osterborg A, Atadja P, Larsson O, Axelson M, Van Camp B, Jernberg-Wiklund H, Vanderkerken K. The HDAC inhibitor LBH589 enhances the antimyeloma effects of the IGF-1RTK inhibitor picropodophyllin. Clin Cancer Res. 2012; 18:2230-2239.

15. Tate CR, Rhodes LV, Segar HC, Driver JL, Pounder FN, Burow ME, Collins-Burow BM. Targeting triple-negative breast cancer cells with the histone deacetylase inhibitor panobinostat. Breast Cancer Res. 2012; 14:R79.

16. Kai M, Kanaya N, Wu SV, Mendez C, Nguyen D, Luu T, Chen S. Targeting breast cancer stem cells in triplenegative breast cancer using a combination of LBH589 and salinomycin. Breast Cancer Res Treat. 2015; 151:281-294.

17. Crisanti MC, Wallace AF, Kapoor V, Vandermeers F, Dowling ML, Pereira LP, Coleman K, Campling BG, Fridlender ZG, Kao GD, Albelda SM. The HDAC inhibitor panobinostat (LBH589) inhibits mesothelioma and lung cancer cells in vitro and in vivo with particular efficacy for small cell lung cancer. Mol Cancer Ther. 2009; 8:2221-2231.

18. Kaneko I, Hazama-Shimada Y, Endo A. Inhibitory effects on lipid metabolism in cultured cells of ML-236B, a potent inhibitor of 3-hydroxy-3-methylglutaryl-coenzyme-A reductase. Eur J Biochem. 1978; 87:313-321.

19. Sirtori CR. The pharmacology of statins. Pharmacol Res. 2014; 88:3-11.

20. Denoyelle C, Vasse M, Korner M, Mishal Z, Ganne F, Vannier JP, Soria J, Soria C. Cerivastatin, an inhibitor of HMG-CoA reductase, inhibits the signaling pathways involved in the invasiveness and metastatic properties of highly invasive breast cancer cell lines: an in vitro study. Carcinogenesis. 2001; 22:1139-1148.

21. Kotamraju S, Williams CL, Kalyanaraman B. Statininduced breast cancer cell death: role of inducible nitric oxide and arginase-dependent pathways. Cancer Res. 2007; 67:7386-7394.

22. Parikh A, Childress C, Deitrick K, Lin Q, Rukstalis D, Yang W. Statin-induced autophagy by inhibition of geranylgeranyl biosynthesis in prostate cancer PC3 cells. Prostate. 2010; 70:971-981. 
23. Zhang Q, Yang YJ, Wang H, Dong QT, Wang TJ, Qian HY, $\mathrm{Xu} \mathrm{H}$. Autophagy activation: a novel mechanism of atorvastatin to protect mesenchymal stem cells from hypoxia and serum deprivation via AMP-activated protein kinase/mammalian target of rapamycin pathway. Stem Cells Dev. 2012; 21:1321-1332.

24. Zhang J, Yang Z, Xie L, Xu L, Xu D, Liu X. Statins, autophagy and cancer metastasis. Int J Biochem Cell Biol. 2013; 45:745-752.

25. Yang Y, Fiskus W, Yong B, Atadja P, Takahashi Y, Pandita TK, Wang HG, Bhalla KN. Acetylated hsp70 and KAP1-mediated Vps34 SUMOylation is required for autophagosome creation in autophagy. Proc Natl Acad Sci USA. 2013; 110:6841-6846.

26. Rao S, Lowe M, Herliczek TW, Keyomarsi K. Lovastatin mediated G1 arrest in normal and tumor breast cells is through inhibition of CDK2 activity and redistribution of p21 and p27, independent of p53. Oncogene. 1998; 17:2393-2402.

27. Motoshima H, Goldstein BJ, Igata M, Araki E. AMPK and cell proliferation--AMPK as a therapeutic target for atherosclerosis and cancer. J Physiol. 2006; 574:63-71.

28. Hardie DG, Alessi DR. LKB1 and AMPK and the cancermetabolism link - ten years after. BMC Biol. 2013; 11:36.

29. Vucicevic L, Misirkic M, Janjetovic K, Vilimanovich U, Sudar E, Isenovic E, Prica M, Harhaji-Trajkovic L, KravicStevovic T, Bumbasirevic V, Trajkovic V. Compound C induces protective autophagy in cancer cells through AMPK inhibition-independent blockade of Akt/mTOR pathway. Autophagy. 2011; 7:40-50.

30. Carew JS, Medina EC, Esquivel JA, 2nd, Mahalingam D, Swords R, Kelly K, Zhang H, Huang P, Mita AC, Mita MM, Giles FJ, Nawrocki ST. Autophagy inhibition enhances vorinostat-induced apoptosis via ubiquitinated protein accumulation. J Cell Mol Med. 2010; 14:2448-2459.

31. Rao R, Balusu R, Fiskus W, Mudunuru U, Venkannagari S, Chauhan L, Smith JE, Hembruff SL, Ha K, Atadja P, Bhalla KN. Combination of pan-histone deacetylase inhibitor and autophagy inhibitor exerts superior efficacy against triple-negative human breast cancer cells. Mol Cancer Ther. 2012; 11:973-983.

32. Di Fazio $\mathrm{P}$, Waldegger $\mathrm{P}$, Jabari $\mathrm{S}$, Lingelbach $\mathrm{S}$, Montalbano R, Ocker M, Slater EP, Bartsch DK, Illig R,
Neureiter D, Wissniowski TT. Autophagy-related cell death by pan-histone deacetylase inhibition in liver cancer. Oncotarget. 2016; 7:28998-29010. doi: 10.18632/ oncotarget.8585.

33. Jager S, Bucci C, Tanida I, Ueno T, Kominami E, Saftig P, Eskelinen EL. Role for Rab7 in maturation of late autophagic vacuoles. J Cell Sci. 2004; 117:4837-4848.

34. Hyttinen JM, Niittykoski M, Salminen A, Kaarniranta K. Maturation of autophagosomes and endosomes: a key role for Rab7. Biochim Biophys Acta. 2013; 1833:503-510.

35. Dawson MA, Kouzarides T. Cancer epigenetics: from mechanism to therapy. Cell. 2012; 150:12-27.

36. Clendening JW, Penn LZ. Targeting tumor cell metabolism with statins. Oncogene. 2012; 31:4967-4978.

37. Araki M, Maeda M, Motojima K. Hydrophobic statins induce autophagy and cell death in human rhabdomyosarcoma cells by depleting geranylgeranyl diphosphate. Eur J Pharmacol. 2012; 674:95-103.

38. Wei YM, Li X, Xu M, Abais JM, Chen Y, Riebling CR, Boini KM, Li PL, Zhang Y. Enhancement of autophagy by simvastatin through inhibition of Rac1-mTOR signaling pathway in coronary arterial myocytes. Cell Physiol Biochem. 2013; 31:925-937.

39. Jiang P, Mukthavaram R, Chao Y, Nomura N, Bharati IS, Fogal V, Pastorino S, Teng D, Cong X, Pingle SC, Kapoor S, Shetty K, Aggrawal A, et al. In vitro and in vivo anticancer effects of mevalonate pathway modulation on human cancer cells. Br J Cancer. 2014; 111:1562-1571.

40. Tricarico PM, Crovella S, Celsi F. Mevalonate Pathway Blockade, Mitochondrial Dysfunction and Autophagy: A Possible Link. Int J Mol Sci. 2015; 16:16067-84.

41. van der Burgh R, Pervolaraki K, Turkenburg $M$, Waterham HR, Frenkel J, Boes M. Unprenylated RhoA contributes to IL-1 beta hypersecretion in mevalonate kinase deficiency model through stimulation of Rac1 activity. J Biol Chem. 2014; 289:27757-27765.

42. Shen HM, Mizushima N. At the end of the autophagic road: an emerging understanding of lysosomal functions in autophagy. Trends Biochem Sci. 2014; 39:61-71.

43. Nara A, Mizushima N, Yamamoto A, Kabeya Y, Ohsumi Y, Yoshimori T. SKD1 AAA ATPase-dependent endosomal transport is involved in autolysosome formation. Cell Struct Funct. 2002; 27:29-37. 